Titre court : Décrochage dans l'enseignement secondaire

\title{
Les déterminants du décrochage dans l'enseignement secondaire : une revue de littérature.
}

The determinants of dropping out in secondary education: a literature review.

Antony Vinciguerra ${ }^{1}$, Isabelle Nanty ${ }^{2}$, Catherine Guillaumin ${ }^{3}$, Emmanuel Rusch $^{34}$, Laurence Cornu ${ }^{3}$, Robert Courtois ${ }^{15}$

${ }^{1}$ Université François Rabelais de Tours, Département de Psychologie, EE 1901 'Qualipsy' (Qualité de vie et Santé psychologique), 37041 Tours cedex, France

2.Université François Rabelais de Tours, Département de Psychologie, EA 2114

'Psychologie des âges de la vie et adaptation', 37041 Tours cedex, France

${ }^{3 .}$ Université François Rabelais de Tours, Département des Sciences de l'éducation, ER 'Education Ethique Santé', 37041 Tours cedex, France

${ }^{4}$ CHRU de Tours, Département de Santé Publique et d'Information médicale, ER 'Education Ethique Santé', Tours, France, 5.CHRU de Tours, CRIAVS 'Centre-Val de Loire'/ Clinique Psychiatrique Universitaire, 37044 Tours cedex, France

Auteur correspondant :

Robert Courtois

Université François Rabelais - Département de Psychologie, 3, rue des tanneurs - BP 4103, F-37041 Tours Cedex 1.

Email : robert.courtois@univ-tours.fr

Nombre de mots : 13590 (6 498 excepté le résumé, l'abstract et les références) 
Antony Vinciguerra est consultant en éducation et formation, doctorant en psychologie à l'Université de Tours. Mots-clés pour les thématiques de recherche : décrochage en études et en formation ; Adolescence et jeunes adultes ; Processus d'autonomisation et de motivation ; Insertion socioprofessionnelle; Ingénierie de formation.

Isabelle Nanty est maître de conférences en psychologie à l'Université de Tours. Mots-clés pour les thématiques de recherche: Clinique développementale; Enfant; Adolescent; Handicap ; Psychopédagogie.

Catherine Guillaumin est maître de conférences en science de l'éducation à l'Université de Tours. Mots-clés pour les thématiques de recherche: Alternance; Réflexivité; Ingénierie ; Coopération; Abandon en formation professionnelle.

Emmanuel Rusch est professeur de santé publique au CHRU de Tours (Université de Tours). Motsclés pour les thématiques de recherche : Education; Parcours de santé ; Economie ; Ethique ; Epidémiologie.

Laurence Cornu est professeur en science de l'éducation à l'Université de Tours. Mots-clés pour les thématiques de recherche : Confiance ; Hospitalité ; Prendre Soin ; Action ; Subjectivation.

Robert Courtois est psychiatre au CHRU de Tours et Maitre de conférences-HDR en psychologie à l'Université de Tours. Mots-clés pour les thématiques de recherche: Violence; Conduites à risque ; Addiction ; Sexualité ; Evaluation de la personnalité. 


\section{Les déterminants du décrochage dans l'enseignement secondaire : une revue de littérature.}

\section{Résumé}

Introduction. Les déterminants individuels du décrochage ont fait l'objet d'importants développements dans la littérature récente. Notre objectif est de faire le point sur les connaissances scientifiques disponibles en ce domaine.

Méthode. L'article présent est une revue de littérature réalisée à partir de PsycInfo et basée sur un total de 50 articles publiés entre 2010 et 2017.

Résultats. Les performances scolaires et les conduites à risque précoces restent les meilleurs déterminants du décrochage. Anxiété, dépression et troubles externalisés peuvent jouer un rôle direct ou indirect dans la prédiction du décrochage. Le manque de soutien psychoaffectif favorise fortement l'intention de décrocher.

Discussion. Les biais méthodologiques nuancent l'importance du genre, des résultats scolaires, et du redoublement. Le développement des connaissances sur les relations entre différents déterminants, les profils d'élèves à risque et les temporalités à risque doivent permettre de mieux comprendre les trajectoires de décrochage et les processus à l'œuvre au sein de différents contextes scolaires.

Mots-clés : Décrochage Scolaire; Enseignement Secondaire ; Adolescence ; Facteurs Individuels ; Facteurs psychopathologiques. 


\section{The determinants of dropping out in secondary education: a literature review.}

\section{Abstract}

Introduction. Dropping out of school is a research topic with various dimensions: nongraduation, school leaving, absenteeism, school failure and school refusal. It involves processes determined by various factors - individual, social, familial and institutional. Individual determinants have specifically been studied in the recent literature. They involve demographic and academic indicators, behaviors, attitudes and mental health of the student, as well as specific types of school refusal. The aim of our study is to update knowledge in this specific field, and highlight underlying problems and methodological issues raised in the literature.

Method. Dropping out of school is defined as leaving school without a qualification. It involves a process of specific disengagement, which is non-reducible to the negative counterpart of school commitment. The present article is a review of literature from PsycInfo on the individual determinants of dropping out of school. It is based on 50 articles published between 2010 and 2017, related to the following topics: school dropouts, school leavers, school refusal, and academic failure. Five exclusion criteria were used: studies with single factor analysis, literature review, sample excluding secondary education, sample of less than $\mathrm{N}=100$, non-general, non-mixed, or composed of a socio-demographic minority.

Results. School performance and early risk behaviors (substance use disorders, sexuality) remain the best determinants of dropping out throughout the duration of schooling. Psychopathological factors, anxiety, depression and externalized disorders can play a direct or indirect role in the prediction of dropping out. Lack of psycho-emotional support strongly supports the intention to drop out. Motivation has an unequal predictive value, depending on the type of construct or theoretical model used. Inappropriate beliefs, low self-esteem, pessimism, creativity appear as indirect determinants of dropping out. School burnout, understood as emotional, physical and mental exhaustion due to education, directly causes a break between the student and the school.

Discussion. Methodological bias can modify the significance of determinants such as gender, academic achievement and retention. Greater knowledge about the relationships between determinants, the profiles of at-risk students and temporalities should help clarify students' trajectories and the processes at work in different school contexts. Recommendations to educational staff are put forward regarding the detection of individual indicators of dropping out.

Key-words: School Dropouts; Secondary School; Adolescence; Individual Factors; Psychopathologic Factors. 


\section{Introduction.}

Le décrochage scolaire est une préoccupation majeure des politiques publiques en matière d'éducation et de formation (Bulletin Officiel n ${ }^{\circ} 6$ du 10 février 2011, 2011; OECD, 2016; Parlement Européen, 2009). En France, les données collectées par le Ministère de l'Éducation nationale, de l'Enseignement supérieur et de la Recherche pour les années 20132014 indiquent que 140000 élèves quittent chaque année le cursus scolaire sans diplôme (baccalauréat, certificat d'aptitude professionnelle, brevet d'études professionnelles ou équivalent) tandis que le nombre de 18-24 ans n'ayant acquis aucun diplôme en formation initiale ou continue s'élève à 620000 (Weixler \& Soudoplatoff, 2015).

Les raisons d'une telle situation sont multiples et complexes. Les données de la littérature en sociologie, sciences de l'éducation et psychologie s'accordent sur la variété des facteurs à l'œuvre dans le décrochage, et sur la nécessité de concevoir celui-ci comme un objet de recherche pluridimensionnel (Attwood, Croll, \& Hamilton, 2005; Fortin \& Picard, 1999; Fortin, Royer, Potvin, Marcotte, \& Yergeau, 2004; Janosz, 2000; Janosz \& Le Blanc, 1997; Jimerson, Egeland, Sroufe, \& Carlson, 2000; Warren \& Lee, 2003). L'efficacité de la prévention et de la résolution du décrochage repose sur une compréhension fine des liens entre mécanismes institutionnels et problématiques psychosociales, afin de pouvoir approcher les trajectoires individuelles de décrochage comme des phénomènes non-linéaires et de contextualiser les réponses appropriées aux situations menant au décrochage scolaire. Les facteurs relevant de l'élève, du contexte familial et du contexte scolaire sont autant de dimensions à prendre en compte et à connecter (Bernard, 2015; Rumberger \& Lim, 2008).

Une série d'études a montré dans cette perspective que les décrocheurs ne constituaient pas de groupes homogènes de profils-types d'élèves en difficulté, mais un ensemble de trajectoires et de situations de désengagement scolaire individuel dans lesquelles des déterminants communs pouvaient être considérés (Archambault, Janosz, Fallu, \& Pagani, 2009; Fortin, Marcotte, Potvin, Royer, \& Joly, 2006; Gélinas et al., 2000; Janosz, Le Blanc, Boulerice, $\&$ Tremblay, 2000). Les phénomènes de temporalités constituent un autre type de dimension à considérer pour identifier en amont de la sortie du cursus d'études, des périodes ou des évènements à risque. Le temps de l'adolescence, comme processus de maturation biologique et psychosociale, est en grande partie celui de l'enseignement secondaire (Eccles \& Roeser, 2011; Wang \& Peck, 2013). Il constitue en soi une période de vie caractérisée par les conduites à risque, dont le décrochage scolaire constitue de fait une composante (Courtois, 2011; Finn, 1989; Daniel Marcelli \& Braconnier, 2008; McLeod, Uemura, \& Rohrman, 2012). Les facteurs 
de risques de décrochage sont eux-mêmes imparfaitement connus, ceux qui ont été identifiés dans la littérature n'expliquent qu'un peu plus de la moitié des décrochages individuels étudiés (Balfanz, Herzog, \& Mac Iver, 2007; Battin-Pearson et al., 2000; Dynarski \& Gleason, 2002; Janosz, Archambault, Morizot, \& Pagani, 2008). Il reste donc nécessaire de prendre en compte la problématique de l'élève dans son caractère le plus singulier, sa dynamique psychique et relationnelle au sein de son environnement.

Notre étude dresse un état des lieux de la littérature psychologique récente (années 20102017) sur les déterminants du décrochage scolaire. Elle a pour objectif de faire le point sur les connaissances scientifiques disponibles sur la valeur des attitudes, comportements et troubles mentaux, dans les processus de décrochage, et d'en tirer des applications pratiques pour la prévention et la résolution du décrochage.

\section{Méthode.}

Identifier la valeur d'un déterminant dans la prédiction du décrochage soulève d'importants problèmes méthodologiques (Battin-Pearson et al., 2000; Berthet \& Zaffran, 2011; Hammond, Linton, Smink, \& Drew, 2007; Kronick \& Hargis, 1998; Lehr, Hansen, Sinclair, \& Christenson, 2003; Rumberger, 1987, 1995; Rumberger \& Lim, 2008; Rumberger \& Palardy, 2004).

\subsection{Définition des dimensions du décrochage.}

Dans le cadre d'une revue de littérature, la principale difficulté méthodologique est la définition de la notion de décrochage elle-même, qui inclut une série d'autres notions parentes, mais non équivalentes (Esterle-Hedibel, 2006; Moulin, Doray, Prévost, \& Delavictoire, 2014). Au sens institutionnel le plus strict, le décrochage scolaire signifie la sortie du cursus avant son terme, c'est-à-dire sans l'obtention d'un diplôme. Lorsqu'il intervient avant 16 ans, il correspond à la notion parente de «déscolarisation », qui possède une dimension institutionnelle et légale : le manquement à la scolarité obligatoire (Geay \& Meunier, 2003; Glasman \& Douat, 2004). Les notions « d'échec » et «d'abandon » renvoient à des dimensions plus spécifiques : l'échec scolaire peut s'employer pour les difficultés d'apprentissage et l'insuccès progressif ou définitif aux évaluations scolaires, tandis que l'abandon scolaire marque une interruption de la scolarité sans volonté manifeste de la reprendre et sans nécessairement avoir échoué à l'obtention d'un diplôme (Moulin et al., 2014). Le «refus scolaire anxieux », parfois appelé «phobie scolaire», est une notion psychopathologique 
spécifique liée aux troubles anxieux (Brandibas, Jeunier, Clanet, \& Fourasté, 2004; GalléTessonneau, Doron, \& Grondin, 2016; King \& Bernstein, 2001; Sibeoni, Moscoso, \& RevahLevy, 2016). Ces types de troubles ont pour conséquence directe une rupture avec l'école, mais ils prennent leur source en dehors du contexte scolaire, dans l'angoisse de séparation, cristallisée notamment lors du passage de l'école primaire au collège, ou plus globalement dans des complications du développement psychique de l'adolescent (D Marcelli \& Mezange, 2000). Il est néanmoins nécessaire de tenir compte de ce type de facteurs dans la prévention du décrochage pour expliquer le déclenchement de comportements scolaires déviants comme l'absentéisme (Kearney, 2008) et pour distinguer ce qui relève d'une prise en charge thérapeutique ou d'une réponse éducative (Catheline, 2007). Le même constat peut être fait pour le «burnout scolaire», qui constitue un syndrome d'épuisement émotionnel et d'inadéquation envers l'école (Parker \& Salmela-Aro, 2011), et se caractérise notamment par une dimension de cynisme définie comme une attitude détachée et indifférente vis-à-vis du travail scolaire, une perte d'intérêt et une incapacité à donner du sens à sa scolarité.

En tant qu'objet d'études, le décrochage se comprend d'une manière plus globale comme un processus de désengagement, qui doit être considéré selon des dynamiques propres et non comme le simple pendant négatif de l'engagement scolaire (Christenson, Reschly, \& Wylie, 2012; Finn \& Zimmer, 2012; Martin, 2012; Wirthwein, Sparfeldt, Pinquart, Wegerer, \& Steinmayr, 2013). La notion d'absentéisme, distincte de celle de décrochage, peut constituer un indicateur comportemental et académique important du désengagement, en termes de temporalité (durée et fréquence) et de valeur (justifiée, injustifiée, choisie, subie, intérieure, extérieure) (Blaya, 2003; Blaya \& Hayden, 2003; Cristofoli, 2013; Kearney \& Graczyk, 2014; Weitzman, Klerman, Lamb, Menary, \& Alpert, 1982).

\subsection{Définition des critères de sélection des articles.}

Afin de réduire ces difficultés, nous avons concentré notre travail sur un corpus d'articles issu d'une littérature relativement homogène, sur les champs disciplinaires couverts par la base de données PsycInfo. Nous avons déterminé notre recherche à partir des mots-clés de «School Dropouts» (décrocheurs scolaires). La difficulté était d'inclure dans ces termes génériques toutes les dimensions possibles du décrochage scolaire (échec, abandon, déscolarisation, absentéisme, refus scolaire, désengagement, etc...) sans inflation du nombre de mots-clés de recherche documentaire et en tenant compte des spécificités sémantiques des termes utilisés dans la langue anglaise. Nous avons recherché dans les indexations de PsycInfo des descripteurs-sujets, c'est-à-dire les concepts transversaux les plus souvent liés au sujet dans 
la littérature, pour obtenir une équation de mots clé qui ne soit ni trop exploratoire ni trop complexe :

- DE («School Dropouts » OR «School Leavers » OR «School Refusal» OR «Academic Failure »)

Nous avons limité nos résultats aux articles parus dans des revues à comités de lecture entre 2010 et 2017 pour obtenir un nombre de 956 articles. Cinq critères d'inclusion ont été employés pour affiner notre base d'articles (voir Figure 1):

- une population d'étude en enseignement secondaire

- une méthode quantitative

- une variable dépendante liée au décrochage

- au moins une variable indépendante liée à un facteur psychologique

- une population d'étude non-générale, non-mixte, ou constituée par une minorité sociodémographique

L'étude porte au final sur 50 articles dont le détail est présenté dans le tableau 1.

S'il vous plait, insérer la figure 1 et le tableau 1 environ ici

\section{Résultats.}

\subsection{Les déterminants démographiques et académiques.}

Les principaux marqueurs d'un décrochage effectif ou d'une trajectoire de décrochage sont les faibles résultats scolaires, le redoublement d'une ou plusieurs années de classe, le fait d'être un garçon - ou du moins de se comporter comme tel.

\subsubsection{La performance scolaire.}

La performance et la réussite scolaire d'un élève est un comportement mesuré par ses résultats aux évaluations scolaires. Son importance est avérée lorsque les résultats scolaires sont analysés seuls, ou en association avec d'autres facteurs, notamment le manque d'engagement, de motivation, ou de satisfaction scolaire (Bowers \& Sprott, 2012a; Casillas et al., 2012; Fortin, Marcotte, Diallo, Potvin, \& Royer, 2013; Hodis, Meyer, McClure, Weir, \& Walkey, 2011; Lessard, Poirier, \& Fortin, 2010). La valeur prédictive des faibles performances scolaires reste importante selon différentes modalités d'évaluation : résultats globaux (niveaux de Grade Point Average - GPA américain) ou moyenne générale (Bardou, Oubrayrie-Roussel, \& Lescarret, 
2012; Casillas et al., 2012; Gagnon et al., 2015; Hawkins, Jaccard, \& Needle, 2013; Jung, Leroux, \& Truscott, 2017), résultats présents et antérieurs (Casillas et al., 2012), résultats dans des matières principales comme les mathématiques et la langue écrite maternelle (Abar, Abar, Lippold, Powers, \& Manning, 2012; Bowers \& Sprott, 2012b; Cornell, Gregory, Huang, \& Fan, 2013; Fan \& Wolters, 2014; Gagné, Marcotte, \& Fortin, 2011; Lamote, van Damme, et al., 2013; Quiroga, Janosz, Bisset, \& Morin, 2013). Les seuls résultats en mathématiques peuvent enfin apparaitre comme principal prédicteur de décrochage (Bowers, Sprott, \& Taff, 2013; Hakkarainen, Holopainen, \& Savolainen, 2015). La performance scolaire possède une dimension cognitive, dans la capacité de l'élève à acquérir du savoir, à le mémoriser, et à le restituer. Nous avons relevé pour cette dimension une étude portant sur l'intelligence fluide (Pagani, Brière, \& Janosz, 2017). Elle montre qu'un faible développement de ce type d'intelligence constitue un déterminant de premier ordre dans l'échec scolaire. Sa valeur de prédiction de décrochage est inférieure à celles d'être un garçon et d'être lié à un milieu familial en difficulté, mais est supérieure à celles des conduites délinquantes et de l'engagement scolaire.

\subsubsection{Le redoublement.}

Les travaux comparatifs de Bowers et al. (2013) montrent que le redoublement est le meilleur prédicteur du décrochage après les résultats scolaires. Il apparait comme déterminant premier lorsqu'il est étudié avec des variables sociodémographiques et académiques selon une approche longitudinale (Lamote, Speybroeck, Van Den Noortgate, \& Van Damme, 2013; Lamote, van Damme, et al., 2013; Quiroga, Janosz, Lyons, \& Morin, 2012) et avec des variables de performance scolaire selon une approche non longitudinale (Casillas et al., 2012; Wood et al., 2017). Il reste déterminant quelle que soit l'année de redoublement, y compris pour un redoublement en primaire (Lamote, van Damme, et al., 2013). Sa corrélation avec une situation de décrochage est particulièrement importante en fin d'enseignement secondaire, notamment en cas de redoublement en classe de $2^{\text {nde }}$ (Pharris-Ciurej, Hirschman, \& Willhoft, 2012) et de $1^{\text {ère }}$ (Abar et al., 2012). Les causes du redoublement peuvent être elles-mêmes expliquées par un désengagement scolaire latent, la présence de troubles psychiques ou la récurrence de comportements déviants ou à risque (Legleye et al., 2010; Quiroga et al., 2012).

\subsubsection{Le genre.}

Le fait d'être un garçon représente un facteur de risque de décrochage couramment établi, même s'il n'apparait pas significatif systématiquement (Blaya \& Fortin, 2011; Borgna 
\& Struffolino, 2017; Delfabbro, Winefield, Winefield, Malvaso, \& Plueckhahn, 2017; Fan \& Kearney, Hendron, \& Schafer, 2011; Kim \& Hull, 2012; Lamote, Speybroeck, et al., 2013; Legleye et al., 2010; Markussen et al., 2011; Pagani et al., 2017; Quiroga et al., 2013; Quiroga et al., 2012; Wood et al., 2017). Une nuance importante à la valeur du facteur genre a été apportée par une étude spécifiquement consacrée à la question (Theunissen, de Man, Verdonk, Bosma, \& Feron, 2015). Elle démontre via l'utilisation d'échelles de mesure des stéréotypes de genre sur un groupe mixte constitué de $50 \%$ de décrocheurs, qu'une adhésion importante à des croyances perçues comme masculines constituait un meilleur prédicteur de décrochage que l'appartenance biologique au sexe masculin.

\subsection{Les facteurs d'attitudes.}

Les facteurs d'attitudes jouent dans le décrochage un rôle inégal du fait de l'hétérogénéité des notions employées pour caractériser ces facteurs. Motivation, autonomie, sens du relationnel ou estime de soi, sont mesurés selon différents modèles et indicateurs d'attitudes directes ou indirectes (croyances sur soi, sur ses habilités, sur les autres, sur l'école, etc...), ce qui rend difficile la comparaison de résultats.

\subsubsection{La motivation.}

Les différentes dimensions de motivation composant le modèle de la théorie de l'autodétermination (Self-Determination Theory - SDT) permettent de définir des typologies d'élèves réussissant leurs études, mais ne peuvent expliquer qu'en partie les trajectoires de décrochage (Abar et al., 2012; Grobler et al., 2014). Abar et al. (2012) ont pu identifier dans un contexte américain que les élèves témoignant d'une motivation unidimensionnelle étaient également ceux qui étaient le plus touchés par le décrochage en fin d'études secondaires, notamment si cette motivation était extrinsèque. Ces résultats ne se retrouvent pas dans l'étude menée dans un contexte sud-africain par Grobler et al. (2014). Considérée via le score global de Self Determination Index - SDI, la motivation scolaire en elle-même n'apparait pas comme un facteur direct de décrochage (Ricard \& Pelletier, 2016). La motivation scolaire définie selon des attitudes directes envers l'école, peut en revanche constituer un facteur indirect de décrochage dans d'autres modèles. Dans le Self-System Model of Motivational Development - SSMMD, l'identification à l'école et le contrôle perçu déterminent deux facteurs comportementaux directs de décrochage, la performance scolaire d'une part, l'engagement dans les études d'autre part (Fall \& Roberts, 2012). Dans l'Expectancy-values Model of Motivation 
- EVMM, les croyances et attitudes négatives des élèves sur leurs capacités scolaires tendent à produire des projets d'études postérieures peu aboutis, qui déterminent directement le décrochage (Fan \& Wolters, 2014). La valeur prédictive du projet d'étude dans le décrochage est toutefois moins importante que celle des indicateurs socio-économiques (revenu familial, appartenance à une minorité ethnique) utilisés comme variables de contrôle.

\subsubsection{Le soutien psychosocial.}

Le soutien psychosocial (support social ou environnemental) est l'un des types de facteurs les plus étudiés dans la littérature récente, sans qu'un consensus puisse être dégagé sur sa valeur. L'étude de Casillas et al. (2012) lui donne une valeur de prédiction de décrochage inférieure à celui des redoublements et des performances scolaires, mais supérieure à ceux des facteurs comportementaux, scolaires et démographiques. Une étude portant sur des élèves norvégiens âgés de 16 ans, montre que le sentiment de solitude est le premier déterminant d'une intention de décrocher, parmi des facteurs de genre, d'évaluations académiques, de support parental et professoral (Frostad, Pijl, \& Mjaavatn, 2015). Le manque d'autonomie relationnelle est dans la littérature l'une des caractéristiques des élèves décrocheurs (Fortin et al., 2013; Frostad et al., 2015; Jung et al., 2017; Lessard, Butler-Kisber, Fortin, \& Marcotte, 2014; Orpinas \& Raczynski, 2016; Orpinas, Raczynski, Peters, Colman, \& Bandalos, 2015). Plus particulièrement la difficulté à savoir nouer des liens d'amitié réciproques constitue un facteur direct de décrochage (Bowers \& Sprott, 2012a; Ricard \& Pelletier, 2016). Les élèves se déclarant à risque de décrochage sont également ceux témoignant de plus de solitude à l'école et du sentiment d'être une victime. Cette solitude peut se traduire par le fait de subir du harcèlement scolaire (Bullying), et entrainer dans ce cas un abandon des études (Cornell et al., 2013). De même, le sentiment de rejet social peut à la fois conduire à des conduites déviantes et à un désengagement vis-à-vis de l'école (Jung et al., 2017). A l'inverse une étude longitudinale prenant en compte 12 facteurs scolaires ne donne aucun rôle direct au soutien des parents ou des professeurs dans les trajectoires de décrochage mesurées à partir de la performance scolaire (Lucio, Hunt, \& Bornovalova, 2012). Le support affectif des parents peut jouer un rôle indirect dans le décrochage s'il vient à manquer dans la satisfaction des besoins psychologiques de base (Lovelace, Reschly, Appleton, \& Lutz, 2014; Ricard \& Pelletier, 2016), ou dans l'intention de réussir ses études (Freeney \& O'Connell, 2012). Une faible communication parents-élève sur le sujet de l'école, associée à une motivation unidimensionnelle pour l'école, peut caractériser une trajectoire de décrochage en fin d'études secondaires (Abar et al., 2012). La valeur du soutien des enseignants comme facteur de décrochage apparait également indirecte via la satisfaction des besoins psychologiques de base, 
les attitudes directes envers l'école ou encore l'intention de décrocher (Bowers et al., 2013; Fan \& Wolters, 2014; Freeney \& O'Connell, 2012; Frostad et al., 2015; Ricard \& Pelletier, 2016).

\subsubsection{Les attitudes négatives et inadaptées.}

Un ensemble de facteurs émotionnels (nervosité, négativité, isolement social, etc...) et d'attitudes directes négatives envers l'école est également identifié dans la littérature (Blaya \& Fortin, 2011; Bowers et al., 2013; Lamote, Speybroeck, et al., 2013; Lovelace et al., 2014; Quiroga et al., 2013; Wang \& Fredricks, 2014). Percevoir peu de sentiments de satisfaction et de réussite peut constituer un facteur de risque de décrochage (Lessard et al., 2010), de même que le manque d'investissement dans les devoirs et de faibles attentes vis-à-vis de l'école (Fan \& Wolters, 2014; Hodis et al., 2011; Lucio et al., 2012). La créativité a pu être posée comme un facteur de décrochage lorsqu'elle était associée à des aspirations professionnelles fortes en fin d'études secondaires (Kim \& Hull, 2012). Ce type d'aspirations se retrouve dans les trajectoires de décrochage associées à des engagements extrascolaires forts en fin d'études secondaires (Bowers \& Sprott, 2012a; Lovelace et al., 2014). Plus directement, le fait de ne pas aimer l'école peut apparaitre comme l'une des principales raisons de quitter celle-ci (Kim \& Hull, 2012). Le manque d'optimisme, l'excès de pessimisme et un fort stress perçu sur la durée de l'année scolaire peuvent également déterminer directement l'intention de décrocher ou des comportements de désengagement (Eicher, Staerklé, \& Clémence, 2014; Meylan, Doudin, Curchod-Ruedi, \& Stephan, 2015). Les difficultés à s'affirmer à l'école et à s'adapter aux contraintes d'études caractérisent d'autres types de facteurs de trajectoires de décrochage (Fortin et al., 2013; Orpinas et al., 2015). Le burnout scolaire compris comme un épuisement émotif, physique et mental dû à la poursuite d'études, peut être directement provoqué par une inadéquation entre les croyances de l'élève et les exigences du cursus scolaire, en termes de perte de contrôle de soi et surtout de sentiment d'inutilité des efforts produits (Lee, Puig, \& Lee, 2012). Considérée globalement sur un suivi longitudinal, l'estime de soi n'apparait pas comme un marqueur caractéristique des élèves décrocheurs (Delfabbro et al., 2017). Elle a pu être associée à des élèves cumulant de mauvaises performances académiques, selon plusieurs dimensions prédictives : une faible estime de soi «socio-émotionnelle » entraine un rapport négatif à l'école et une démobilisation scolaire globale, tandis qu'une faible estime de soi « scolaire » prédit une démobilisation dans les apprentissages (Bardou et al., 2012). Le rôle d'une dimension de l'estime de soi «physique » (rapport à l'image corporelle personnelle) reste relatif dans les comportements de désengagement scolaire (Bardou et al., 2012; Ingul \& Nordahl, 2013). La mauvaise santé physique apparait en revanche comme un facteur de décrochage (Delfabbro et al., 2017), tandis que le manque latent de maturité dans le soin 
accordé à son corps et à sa santé est déterminant lorsqu'il est associé au trait de personnalité d'introversion (Migali \& Zucchelli, 2017).

\subsection{Les troubles mentaux.}

Les troubles mentaux peuvent se classer entre troubles internalisés (états anxieux et dépressifs) et externalisés (troubles des conduites, troubles oppositionnels avec provocation et personnalité antisociale).

\subsubsection{Les états anxieux.}

Certains troubles particuliers apparaissent particulièrement présents chez les élèves décrocheurs : les troubles du déficit de l'attention et de l'hyperactivité combinée (Blaya \& Fortin, 2011; Breslau, Miller, Chung, \& Schweitzer, 2011). Ces facteurs n'expliquent toutefois pas toute la variance du phénomène de décrochage. Les difficultés d'apprentissage causées par le manque d'attention et les difficultés d'intégration scolaire liées à un relationnel conflictuel pourraient jouer le rôle d'un facteur médiateur avec le décrochage (Breslau et al., 2011). L'absentéisme peut également avoir ce rôle en tant que comportement causé par des facteurs d'anxiété (Ingul, Klöckner, Silverman, \& Nordahl, 2012; Ingul \& Nordahl, 2013). L'anxiété sociale et l'anxiété de séparation produisent en particulier des comportements d'évitement de situations scolaires anxiogènes et la recherche de figures d'attachement à l'extérieur de l'école (Haight et al., 2011). Les problèmes de cognition et notamment les pensées automatiques négatives sur les difficultés scolaires personnelles, apparaissent enfin comme un facteur de refus scolaire anxieux (Maric, Heyne, MacKinnon, van Widenfelt, \& Westenberg, 2013).

\subsubsection{Les états dépressifs.}

La dépression peut également produire un évitement ou une fuite de tout engagement scolaire (Haight et al., 2011). Une série d'études canadiennes a montré sur ce point un effet de type «cumulatif », dans lequel l'état dépressif apparait comme déterminant d'un abandon d'études au terme d'un processus de décrochage caractérisé par d'autres facteurs. La dépression peut apparaitre comme la principale variable modératrice entre les difficultés scolaires rencontrées en primaire et les situations de décrochage constatées dans le secondaire (Quiroga et al., 2012). Elle peut également déterminer indirectement le décrochage via la sensation de ne pas avoir la maitrise ou le contrôle de ses capacités scolaires (Quiroga et al., 2013). D’autres résultats montrent que la dépression peut jouer un rôle négatif décisif dans le basculement des trajectoires scolaires vers le décrochage en fin d'études secondaires pour les élèves cumulant 
chroniquement de mauvais résultats (Gagné et al., 2011). Une dernière étude longitudinale décrocheurs : aux faibles résultats scolaires se cumulent des états dépressifs associés à des facteurs psychosociaux (relations avec la famille, les enseignants et les pairs) (Fortin et al., 2013).

\subsubsection{Les troubles externalisés.}

Les troubles externalisés peuvent exercer un rôle indirect sur le décrochage en favorisant dans un premier temps l'absentéisme scolaire (Ingul et al., 2012; Ingul \& Nordahl, 2013), notamment dans le cas de troubles d'opposition associés à la recherche d'un renforcement personnel hors de l'école (Haight et al., 2011). Ce dernier cas engage souvent une fuite à la fois de l'école et du foyer familial, et in fine à une rupture définitive avec les études (Knollmann, Reissner, Kiessling, \& Hebebrand, 2013). Les troubles externalisés peuvent enfin apparaitre comme des prédicteurs directs de décrochage lorsqu'ils se cumulent à des troubles internalisés sur un suivi longitudinal ou non (Knollmann et al., 2013; Orpinas et al., 2015). Breslau et al. (2011) ont montré via une étude rétrospective sur des facteurs de santé mentale et de conduites à risque, menée à grande échelle (échantillon de près de 30000 personnes) qu'un nombre réduit de troubles externalisés spécifiques pouvait expliquer seul, un décrochage : les troubles des conduites et les différents types de trouble du déficit de l'attention avec ou sans hyperactivité (TDAH). Ceux-ci pourraient jouer un rôle décisif dans la rupture avec l'école en produisant d'une part de mauvais résultats scolaires à cause de problèmes d'attention et de mémoire, d'autre part des problèmes de discipline et de perte de motivation scolaire dus à de fréquents conflits interpersonnels au sein de l'école.

\subsection{Les conduites à risque adolescentes.}

La notion de conduites à risque est associée ici à l'adolescence car elle concerne en grande partie des attitudes et des comportements dont la précocité représente à terme un risque pour la santé. Elle inclut les consommations de tabac, d'alcool, de cannabis, et la sexualité à risque. Celles-ci peuvent être comprises dans la catégorie des troubles mentaux en tant que comportements liés à des problèmes de santé mentale. Nous les avons traités à part pour nous concentrer sur leurs caractéristiques (âge, fréquence).

\subsubsection{La consommation de tabac.}

La consommation précoce de tabac est, parmi les types de consommation de substances à risque, le facteur le plus important de décrochage (Breslau et al., 2011; Hawkins et al., 2013). 
Son rôle dans les processus de décrochage apparait lié au développement de conduites déviantes (Hawkins et al., 2013).

\subsubsection{La consommation de cannabis.}

La consommation globale de cannabis prédit fortement le décrochage lorsqu'associée à la consommation régulière de tabac ou d'alcool (Delfabbro et al., 2017; Legleye et al., 2010; Wang \& Fredricks, 2014). Cette valeur prédictive est renforcée par le fait d'avoir des parents sans emploi ou de faible qualification et d'avoir redoublé à l'école primaire (Legleye et al., 2010), ou encore par un état émotif fragile (Wang \& Fredricks, 2014). Le rôle de la consommation de cannabis dans le décrochage prend une valeur plus faible que celle des troubles de l'anxiété lorsque ces variables sont étudiées selon une approche longitudinale (Ingul et al., 2012). Certains résultats montrent par ailleurs que seul un usage commencé avant 16 ans, à 15 ans voire avant 14 ans, possède un lien statistique significatif avec un décrochage en fin d'études secondaires (Legleye et al., 2010). D'autres indiquent que le fait d'avoir expérimenté du cannabis sans consommation régulière n'a pas de valeur prédictive particulière pour le décrochage (Breslau et al., 2011).

\subsubsection{La consommation d'alcool.}

La consommation d'alcool est en général mesurée en même temps que la consommation d'autres substances psychoactives. Sa valeur prédictive de décrochage est relative, notamment en comparaison de celle de la consommation de tabac, de cannabis et de drogues dures (Breslau et al., 2011; Legleye et al., 2010; Wang \& Fredricks, 2014). La consommation fréquente et précoce d'alcool ne favorise pas la réussite scolaire mais n'apparait pas clairement comme un déterminant direct de décrochage (Breslau et al., 2011; Legleye et al., 2010).

\subsubsection{Les rapports affectifs et sexuels précoces.}

Nous avons relevé dans Hawkins et al. (2013) des résultats donnant l'activité sexuelle précoce comme prédicteur de décrochage en association avec la consommation précoce de tabac. Ils sont à mettre en lien avec ceux trouvés par Orpinas et al. (2013), qui montrent que le fait d'avoir des relations de couple précoces associé à une consommation de tabac, d'alcool ou de cannabis, favorise fortement le décrochage.

\subsubsection{Les conduites déviantes.}

L'étude de Jung et al. (2017) se concentre sur les conduites déviantes en termes de conduites agressives. Elle permet de distinguer l'agressivité réactive et l'agressivité proactive comme deux construits distincts liés à des facteurs de risques spécifiques. La première est 
associée au rejet social, tandis que la seconde engage directement des trajectoires de décrochage par la fréquentation de pairs ayant eux-mêmes des conduites déviantes. Le fait de fréquenter d'autres élèves en difficultés et de témoigner soi-même d'un comportement déviant est également mis en valeur comme facteur de décrochage dans l'étude de Quiroga et al. (2012).

\section{Discussion.}

\subsection{Apports de la littérature récente.}

La littérature récente confirme les connaissances acquises sur les déterminants du décrochage scolaire dans l'enseignement secondaire, et précise leur rôle dans les processus à l'œuvre. La performance scolaire apparait comme le marqueur le plus fiable d'un processus de décrochage (Bowers et al., 2013; De Witte, Cabus, Thyssen, Groot, \& van den Brink, 2013). Des nuances méthodologiques doivent toutefois être apportées à l'importance des résultats scolaires pour mesurer cette performance, car ceux-ci peuvent être biaisés par la provenance des données, rapportée depuis les dossiers scolaires académiques ou auto-rapportée(Gagnon et al., 2015), et également par le type de suivi longitudinal et quantitatif, qui peut confondre décrochage et changement d'établissement pour des élèves cumulant de mauvais résultats. Une meilleure connaissance des facteurs d'intelligence impliqués dans la performance scolaire, notamment dans le rôle médiateur avec l'engagement en études (Pagani et al., 2017) pourrait permettre de mieux évaluer l'incidence des capacités cognitives des élèves. L'impact du redoublement doit également être nuancé, en termes de conséquences individuelles et éducatives (Goos et al., 2013). Les redoublants sont jusqu'à 5 fois plus nombreux que les nonredoublants à décrocher (Quiroga et al., 2012), mais plus que le fait d'avoir cumulé au moins deux fois la même classe, celui d'être plus âgé que la moyenne serait le facteur direct de décrochage, notamment en fin d'études secondaires et lors de l'accès à la majorité (De Witte et al., 2013; Delfabbro et al., 2017; Wood et al., 2017).

Le genre masculin reste dans la littérature un des principaux déterminants du décrochage. Les garçons sont jusqu'à 2 fois et demi plus nombreux que les filles à décrocher (Hodis et al., 2011). Mais certains résultats montrent que le genre masculin peut être considéré comme une variable psychosociale. Le fait de se comporter en garçon peut être influencé par des dimensions de personnalité de type masculine (Theunissen et al., 2015) et des processus de socialisation parentaux et scolaires (Borgna \& Struffolino, 2017). Les liens entre genre masculin et des facteurs spécifiques comme les troubles mentaux restent par ailleurs sujet à 
débat (Esch et al., 2014; Melkevik, Nilsen, Evensen, Reneflot, \& Mykletun, 2016; Quiroga et al., 2012). Le rôle de ces derniers dans le déclenchement de trajectoires de décrochage, est une des principales problématiques de la littérature, notamment en termes d'anxiété et de dépression (Inglés, Gonzálvez-Maciá, García-Fernández, Vicent, \& Martínez-Monteagudo, 2015; Melkevik et al., 2016). Ils constituent en dehors des performances scolaires, le meilleur marqueur des processus longs de décrochage (De Witte et al., 2013) mais sont difficiles à évaluer systématiquement du fait des difficultés à opérer des suivis longitudinaux d'élèves sur ce type de facteurs (Breslau et al., 2011; Esch et al., 2014). Les élèves touchés par des troubles des conduites ou un trouble de déficit de l'attention / hyperactivité ont presque deux fois plus de chance de décrocher que les autres, de même que ceux produisant des comportements déviants (Breslau et al., 2011). Les études indiquent que les troubles externalisés ont une valeur de prédiction de décrochage supérieure à celle des troubles internalisés, mais ne permettent pas de distinguer si ceux-ci constituent des covariables indépendantes ou s'il existe entre eux un rôle de médiation vers le décrochage (Breslau et al., 2011; Esch et al., 2014; Melkevik et al., 2016). L'étude de Quiroga et al. (2012) indiquent que les élèves qui présentent des symptômes de dépression au début du cursus d'enseignement secondaire ont entre 2 et 3 fois plus de chance de décrocher par la suite. D'autres études avancent un effet de cumul entre facteurs psychopathologiques et d'autres types de facteurs, qui joue notamment entre dépression et résultats scolaires (Fortin et al., 2013; Gagné et al., 2011). Les troubles externalisés auraient un impact plus direct en produisant des comportements déviants et perturbateurs à l'école qui déterminent le décrochage (Breslau et al., 2011; Esch et al., 2014). Les troubles internalisés sont par ailleurs fortement liés au burnout scolaire (Walburg, 2014). Le rôle de l'anxiété de séparation peut s'expliquer par la difficulté de l'élève à suivre son cursus à cause de l'angoisse ressentie à l'extérieur du foyer familial et l'entretien de la croyance que ses parents vont tomber malades ou se séparer s'il ne reste pas prêt d'eux. Les facteurs d'éducation et de soutien parental, notamment l'absence d'un cadre de règles claires favorisent fortement ce type de croyances et par conséquent le décrochage.

L'apport principal de la littérature sur les conduites à risque concerne la forte valeur prédictive des conduites précoces (c'est-à-dire qui débutent dès l'entrée au collège) pour le décrochage, notamment pour la consommation de tabac et les relations intimes. Elle permet également de nuancer les effets d'une consommation tardive de cannabis - c'est-à-dire sur les dernières années de la scolarité (De Witte et al., 2013). La relation de la précocité avec la rupture effective avec l'école reste toutefois à préciser. La précocité d'une consommation de tabac ou d'une sexualité à risque peut être la cause ou la conséquence de conduites en décalage avec le 
niveau de maturité psychique et biologique de l'élève. Une hypothèse est que cette académique et social, en renforçant notamment les conduites déviantes à l'extérieur de l'école. La fréquentation de pairs partageant ces conduites pourra produire un renforcement de cellesci, avec le risque que les valeurs de groupe dominent et remplacent les valeurs de l'école (Kindermann, 1993; Liu, Zhao, Chen, Falk, \& Albarracín, 2017; Ryan, 2000, 2001; Wentzel, 1998).

Le soutien psychosocial joue également un rôle négatif dans le vécu subjectif des études lorsqu'il n'est pas trouvé par l'élève auprès de ses parents ou de ses enseignants. Nous avons relevé dans une série d'études que la non-satisfaction des besoins psychologiques de base, le sentiment de solitude, le repli sur soi, le rejet social, l'introversion, le manque de maturité et les croyances négatives sur soi ou sur l'école, favorisent l'intention de décrocher, la démobilisation, voire le burnout scolaire. Le soutien psychosocial et affectif apparait donc comme un déterminant médiateur entre des attitudes et des comportements à risque de décrochage et le développement de comportements de rupture vis-à-vis de l'école, pour un décrochage de l' « intérieur » ou de 1' « extérieur » (Bonnéry, 2004; Catheline, 2007).

\subsection{Limites de l'étude.}

Cette étude est limitée par le traitement non-exhaustif de tous les déterminants connus du décrochage. Elle ne prend pas en compte les travaux concernant les déterminants de décrochage au sein de communautés particulières, de classes sociales défavorisées ou de minorités dites « ethniques ». Plus globalement, elle n'a pas investi le champs des déterminants sociaux et scolaires, qui constituent des prédicteurs de décrochage de premier ordre et des variables de contrôle pour les déterminants individuels (Bernard, 2015; Meuret \& Morlaix, 2006).

L'étude est également limitée par la variété de la littérature, malgré l'utilisation de la base de données PsychInfo. La variable dépendante caractérisant le décrochage est elle-même inégale selon les études. Le dénominateur commun du décrochage reste la sortie du cursus scolaire avant son terme et la non-obtention d'un diplôme. Des critères supplémentaires lui sont associés selon les études, notamment un critère d'âge qui représente le seuil temporel à partir duquel l'arrêt des études peut être considéré comme définitif dans le cadre du système de formation initiale : 17 ans avec une déscolarisation (Legleye et al., 2010), 19 ans avec un cumul d'années d'enseignement scolaire inférieur à 12 (Breslau et al., 2011) ou sans autre indication 
(Fortin et al., 2013), 20 ans (Bowers \& Sprott, 2012a, 2012b; Gagné et al., 2011), ou 21 ans

(Hakkarainen et al., 2015; Wood et al., 2017). Un autre point de nuance se trouve dans la nature de la sortie d'études. Cette dernière peut être définie a minima lorsqu'est constatée une nonréinscription au cursus d'origine, notamment pour l'année de l'obtention du diplôme d'enseignement secondaire. Mais définir une variable dépendante de décrochage sur cette seule base ne prend pas en compte les changements d'établissement et/ou de cursus, ainsi que les reprises d'études après une période d'abandon. Les enquêtes longitudinales nationales les plus complètes fournissent des données susceptibles de réduire ces biais par le croisement des données par établissements : réinscription dans un autre établissement, passage d'un autre diplôme (Orpinas et al., 2013). La conduite anonyme de certaines enquêtes empêche toutefois l'étude longitudinale des trajectoires individuelles (Cornell et al., 2013). D'autres variables à prendre en compte existent comme les situations particulières de décès ou de déménagement dans un autre pays (Abar et al., 2012), mais restent difficiles à contrôler du fait de la difficulté d'accès à ce type de données. Enfin, une partie des études ne prend pas en compte directement les populations de décrocheurs, mais les élèves à risque de décrochage selon différents critères : résultats scolaires (Bardou et al., 2012; Blaya \& Fortin, 2011; Casillas et al., 2012; Hodis et al., 2011; Lessard et al., 2014; Lucio et al., 2012; Meylan et al., 2015), absentéisme récurrent (Haight et al., 2011; Ingul et al., 2012; Ingul \& Nordahl, 2013; Meylan et al., 2015) intention de décrocher (Eicher et al., 2014; Freeney \& O'Connell, 2012; Frostad et al., 2015), symptômes de burnout (Lee et al., 2012), temporalités scolaires à risque (Hakkarainen et al., 2015; Lamote, van Damme, et al., 2013; Pharris-Ciurej et al., 2012).

A ces complications se superposent les difficultés de comparaison objective des résultats d'enquêtes et la nature inégale des méthodologies en termes d'approches et d'analyses statistiques. La nature des facteurs considérés sous un même intitulé peut également être difficile à comparer entre données empiriques ou construits validés, données auto-rapportées ou administratives. La comparaison des résultats peut toutefois être développée en termes de typologies d'élèves à risque et de temporalités à risque.

\subsection{Implications pratiques.}

La littérature précise des temporalités à risque de décrochage, c'est-à-dire des périodes de la scolarité dont les caractéristiques peuvent favoriser une trajectoire de décrochage. L'enseignement primaire constitue une première temporalité à risque. Les premières difficultés métacognitives rencontrées peuvent générer des processus longs favorisant à terme un décrochage, notamment en matière d'états dépressifs ou de «malentendu sociocognitif » 
(Bélanger \& Marcotte, 2013; Lanson \& Marcotte, 2012; Richard \& Marcotte, 2013). L'entrée nouvelle expérience scolaire à laquelle il faut s'adapter, mais également en tant que période correspondant au déclenchement de la puberté (Gagné et al., 2011; Quiroga et al., 2013). Le rôle des parents et des équipes éducatives dans le soutien relationnel aux élèves apparait primordial sur ces premiers temps de scolarité secondaire (Lessard et al., 2014). Les dernières années de l'enseignement secondaire apparaissent comme une temporalité à risque spécifique (Lamote, van Damme, et al., 2013) qui cumule le développement de conduites à risque adolescentes avec les premières élaborations de projets socioprofessionnels extrascolaires. Plus globalement, tout changement de contexte scolaire (changement d'établissement, d'année ou de section) et toute période post-transitoire peuvent constituer des facteurs de risques de décrochage en tant que source potentielle de renforcement des fragilités ou des difficultés préexistantes, notamment des troubles de l'anxiété et de la dépression (Gaspard, Liengme, \& Minjard, 2015; Inglés et al., 2015).

De manière empirique, les données relevées dans cette étude permettent certaines comparaisons avec les composants des typologies de Janosz et al. (2000) et Fortin et al. (2006). Les résultats de Bowers et Sprott (2012b) confirment la pertinence d'une typologie tripartite d'élèves décrocheurs entre les «Désengagés » (cumul de tout type de facteurs, notamment faibles résultats scolaires et investissements scolaires en général, absentéisme, comportements déviants et troubles mentaux), les «Calmes» (cumul de faibles résultats scolaires et d'absentéisme, sans problème particulier de comportements et d'attitudes) et les « Impliqués » (présence d'au moins un facteur d'attitude très marqué, sans autre problème particulier). Ce dernier profil, difficile à prévenir, est à rapprocher de ceux établis sur la base de facteurs sociaux et d'attitude , comme les décrocheurs sans difficultés scolaires apparentes mais en difficulté sociale à l'extérieur de l'école (Bowers \& Sprott, 2012a), les décrocheurs à la motivation scolaire unidimensionnelle (Abar et al., 2012; Hodis et al., 2011; Lovelace et al., 2014), et les décrocheurs à faible estime de soi (Bardou et al., 2012). Les profils identifiés par Knollmann et al. (2013) recoupent les dimensions du SRAS-R (Haight et al., 2011), avec l'anxiété de séparation (rester à la maison avec ses parents) et le trouble de l'opposition (école buissonnière). Les échelles de mesure de ces profils peuvent constituer autant d'outils de prévention des difficultés des élèves dans leur parcours scolaire.

Absence de conflit d'intérêt 


\section{Références bibliographiques.}

Abar, B., Abar, C. C., Lippold, M., Powers, C. J., \& Manning, A. E. (2012). Associations between reasons to attend and late high school dropout. Learning and Individual Differences, 22(6), 856-861. doi:10.1016/j.lindif.2012.05.009

Archambault, I., Janosz, M., Fallu, J.-S., \& Pagani, L. S. (2009). Student engagement and its relationship with early high school dropout. Journal of Adolescence, 32(3), 651-670. doi:10.1016/j.adolescence.2008.06.007

Attwood, G., Croll, P., \& Hamilton, J. (2005). Recovering potential: Factors associated with success in engaging challenging students with alternative pre-16 provision. Educational Research, 47(2), 149-162. doi:10.1080/00131880500104259

Balfanz, R., Herzog, L., \& Mac Iver, D. J. (2007). Preventing student disengagement and keeping students on the graduation path in urban middle-grades schools: Early identification and effective interventions. Educational Psychologist, 42(4), 223-235. doi:10.1080/00461520701621079

Bardou, E., Oubrayrie-Roussel, N., \& Lescarret, O. (2012). Estime de soi et démobilisation scolaire des adolescents. Neuropsychiatrie de l'Enfance et de l'Adolescence, 60(6), 435440. doi:10.1016/j.neurenf.2012.07.003

Battin-Pearson, S., Newcomb, M. D., Abbott, R. D., Hill, K. G., Catalano, R. F., \& Hawkins, J. D. (2000). Predictors of early high school dropout: a test of five theories. Journal of Educational Psychology, 92(3), 568-582. doi:10.1037/0022-0663.92.3.568

Bélanger, M., \& Marcotte, D. (2013). Étude longitudinale du lien entre les changements vécus durant la transition primaire-secondaire et les symptômes dépressifs des adolescents.

Canadian Journal of Behavioural Science/Revue canadienne des sciences du comportement, 45(2), 159-172.

Bernard, P.-Y. (2015). Le décrochage scolaire: «Que sais-je?» $n^{\circ} 3928$ : Presses Universitaires de France.

Berthet, T., \& Zaffran, J. (2011). Le décrochage scolaire: enjeux, acteurs et politiques de lutte contre la déscolarisation. Paper presented at the Le décrochage scolaire: enjeux, acteurs et politiques de lutte contre la déscolarisation: Séminaire du Centre Émile Durkheim qui s' est déroulé en 2011-2012.

Blaya, C. (2003). Absentéisme des élèves : recherches internationales et politiques de prevention.

Blaya, C., \& Fortin, L. (2011). Les élèves français et québécois à risque de décrochage scolaire: Comparaison entre les facteurs de risque personnels, familiaux et scolaires. Orientation Scolaire et Professionnelle, 40(1), 55-85. doi:10.4000/osp.2988

Blaya, C., \& Hayden, C. (2003). Constructions sociales des absentéismes et des décrochages scolaires en France et en Angleterre. Bordeaux, FR: LARSEF/Observatoire européen de la violence scolaire, Université Victor Segalen.

Bonnéry, S. (2004). Décrochage cognitif et décrochage scolaire. In D. Glasman \& F. Euvrard (Eds.), La déscolarisation (pp. 135-149). Paris, FR: La Dispute.

Borgna, C., \& Struffolino, E. (2017). Pushed or pulled ? Girls and boys facing early school leaving risk in Italy. Social Science Research, 61, 298-313. doi:10.1016/j.ssresearch.2016.06.021

Bowers, A. J., \& Sprott, R. (2012a). Examining the multiple trajectories associated with dropping out of high school: A growth mixture model analysis. The Journal of Educational Research, 105(3), 176-195. doi:10.1080/00220671.2011.552075 
Bowers, A. J., \& Sprott, R. (2012b). Why tenth graders fail to finish high school: A dropout typology latent class analysis. Journal of Education for Students Placed at Risk, 17(3), 129-148. doi:10.1080/10824669.2012.692071

Bowers, A. J., Sprott, R., \& Taff, S. A. (2013). Do we know who will drop out? A review of the predictors of dropping out of high school: Precision, sensitivity, and specificity. The High School Journal, 96(2), 77-100. doi:10.7916/D86W9N4X

Brandibas, G., Jeunier, B., Clanet, C., \& Fourasté, R. (2004). Truancy, school refusal and anxiety. School Psychology International, 25(1), 117-126. doi:10.1177/0143034304036299

Breslau, J., Miller, E., Chung, W.-J. J., \& Schweitzer, J. B. (2011). Childhood and adolescent onset psychiatric disorders, substance use, and failure to graduate high school on time. Journal of Psychiatric Research, 45(3), 295-301. doi:10.1016/j.jpsychires.2010.06.014

Bulletin Officiel $n^{\circ} 6$ du 10 février 2011. (2011). Lutte contre le décrochage scolaire, Organisation et mise en œuvre des articles L. 313-7 et L. 313-8 du code de l'Éducation. Paris.

Casillas, A., Robbins, S., Allen, J., Kuo, Y.-L., Hanson, M. A., \& Schmeiser, C. (2012). Predicting early academic failure in high school from prior academic achievement, psychosocial characteristics, and behavior. Journal of Educational Psychology, 104(2), 407-420. doi:10.1037/a0027180

Catheline, N. (2007). Psychopathologie de la scolarité: de la maternelle à l'université (3 ed.). Paris: Elsevier Masson.

Christenson, S. L., Reschly, A. L., \& Wylie, C. (2012). Handbook of research on student engagement. New York, NY, US: Springer Science + Business Media.

Cornell, D., Gregory, A., Huang, F., \& Fan, X. (2013). Perceived prevalence of teasing and bullying predicts high school dropout rates. Journal of Educational Psychology, 105(1), 138-149. doi:10.1037/a0030416

Courtois, R. (2011). Les conduites à risque à l'adolescence. Repérer, prévenir et prendre en charge. Paris, FR: Dunod.

Cristofoli, S. (2013). L'absentéisme des élèves dans le second degré public en 2011-2012. Direction de l'évaluation de la prospective et de la performance Retrieved from http://cache.media.education.gouv.fr/file/2013/07/3/DEPP-NI-2013-01-absenteismeeleves-second-degre-public_242073.pdf.

De Witte, K., Cabus, S., Thyssen, G., Groot, W., \& van den Brink, H. M. (2013). A critical review of the literature on school dropout. Educational Research Review, 10(0), 13-28. doi:10.1016/j.edurev.2013.05.002

Delfabbro, P., Winefield, H., Winefield, A., Malvaso, C., \& Plueckhahn, T. (2017). Factors Associated With Attrition in a 10-year Longitudinal Study of Young People: Implications for Studies of Employment in School Leavers. Australian Psychologist, 52(1), 41-51. doi:10.1111/ap.12207

Dynarski, M., \& Gleason, P. (2002). How can we help? What we have learned from recent federal dropout prevention evaluations. Journal of Education for Students Students Placed At Risk, 7(1), 43-69. doi:10.1097/01.ccx.0000144765.73540.89

Eccles, J. S., \& Roeser, R. W. (2011). Schools as Developmental Contexts During Adolescence. Journal of Research on Adolescence, 21(1), 225-241. doi:10.1111/j.15327795.2010.00725.x

Eicher, V., Staerklé, C., \& Clémence, A. (2014). I want to quit education: A longitudinal study of stress and optimism as predictors of school dropout intention. Journal of Adolescence, 37(7), 1021-1030. doi:10.1016/j.adolescence.2014.07.007 
Esch, P., Bocquet, V., Pull, C., Couffignal, S., Lehnert, T., Graas, M., . . Ansseau, M. (2014). The downward spiral of mental disorders and educational attainment: a systematic review on early school leaving. BMC Psychiatry, 14(1), 237-250.

Esterle-Hedibel, M. (2006). Absentéisme, déscolarisation, décrochage scolaire, les apports des recherches récentes. Déviance et Société, 30(1), 41-65. doi:10.3917/ds.301.0041

Fall, A.-M., \& Roberts, G. (2012). High school dropouts: Interactions between social context, self-perceptions, school engagement, and student dropout. Journal of Adolescence, 35(4), 787-798. doi:10.1016/j.adolescence.2011.11.004

Fan, W., \& Wolters, C. A. (2014). School motivation and high school dropout: The mediating role of educational expectation. British Journal of Educational Psychology, 84(1), 2239. doi:10.1111/bjep.12002

Finn, J. D. (1989). Withdrawing from school. Review of Educational Research, 59(2), 117142. doi:10.3102/00346543059002117

Finn, J. D., \& Zimmer, K. S. (2012). Student engagement: What is it? Why does it matter? In S. L. Christenson, A. L. Reschly, \& C. Wylie (Eds.), Handbook of research on student engagement. (pp. 97-131). New York, NY, US: Springer Science + Business Media.

Fortin, L., Marcotte, D., Diallo, T., Potvin, P., \& Royer, É. (2013). A multidimensional model of school dropout from an 8-year longitudinal study in a general high school population. European Journal of Psychology of Education, 28(2), 563-583. doi:10.1007/s10212012-0129-2

Fortin, L., Marcotte, D., Potvin, P., Royer, E., \& Joly, J. (2006). Typology of students at risk of dropping out of school: description by personal, family and school factors. European Journal of Psychology of Education, 21(4), 363-383. doi:10.1007/BF03173508

Fortin, L., \& Picard, Y. (1999). Les élèves à risque de décrochage scolaire : facteurs discriminants entre décrocheurs et persévérants. Revue des sciences de l'éducation, 25(2), 359-374. doi:10.7202/032005ar

Fortin, L., Royer, É., Potvin, P., Marcotte, D., \& Yergeau, É. (2004). La prediction du risque de decrochage scolaire au secondaire : facteurs personnels, familiaux et scolaires. Canadian Journal of Behavioural Science/Revue canadienne des sciences du comportement, 36(3), 219-231. doi:10.1037/h0087232

Freeney, Y., \& O'Connell, M. (2012). The predictors of the intention to leave school early among a representative sample of Irish second-level students. British Educational Research Journal, 38(4), 557-574. doi:10.1080/01411926.2011.563838

Frostad, P., Pijl, S. J., \& Mjaavatn, P. E. (2015). Losing all interest in school: Social participation as a predictor of the intention to leave upper secondary school early. Scandinavian Journal of Educational Research, 59(1), 110-122. doi:10.1080/00313831.2014.904420

Gagné, M.-E., Marcotte, D., \& Fortin, L. (2011). L'impact de la dépression et de l'expérience scolaire sur le décrochage scolaire des adolescents. The impact of depression and the experience of school dropout on adolescent students. Canadian Journal of Education, 34(2), 72-92.

Gagnon, V., Dupéré, V., Dion, E., Léveillé, F., St-Pierre, M., Archambault, I., \& Janosz, M. (2015). Dépistage du décrochage scolaire à l'aide d'informations administratives ou auto-rapportées. Screening of secondary school dropouts using administrative or selfreported information. Canadian Journal of Behavioural Science / Revue canadienne des sciences du comportement, 47(3), 236-240. doi:10.1037/cbs0000014

Gallé-Tessonneau, M., Doron, J., \& Grondin, O. (2016). Des critères de repérage aux stratégies de prise en charge du refus scolaire: une revue de littérature internationale systématique. Pratiques psychologiques, in press. 
Gaspard, J. L., Liengme, N., \& Minjard, R. (2015). Enjeux et perspectives

psychopathologiques de la phobie scolaire. $=$ Psychopathological problems and viewpoints about school phobia. Neuropsychiatrie de l'Enfance et de l'Adolescence, 63(2), 67-75. doi:10.1016/j.neurenf.2014.11.004

Geay, B., \& Meunier, A. (2003). Enjeux et usages de la "déscolarisation". Cahiers de la recherche sur l'éducation et les savoirs(2), 7-19.

Gélinas, I., Potvin, P., Marcotte, D., Fortin, L., Royer, E., \& Leclerc, D. (2000). Étude des liens entre le risque d'abandon scolaire, les stratégies d'adaptation, le rendement scolaire et les habiletés scolaires. Revue canadienne de psycho-éducation, 29(2), 223-240.

Glasman, D., \& Douat, E. (2004). Qu'est-ce que la déscolarisation ? In D. Glasman \& F. (Euvrard (Eds.), La déscolarisation (pp. 13-69). Paris, FR: La Dispute.

Goos, M., Belfi, B., de Fraine, B., van Damme, J., Onghena, P., \& Petry, K. (2013). Effecten van zittenblijven in het basis- en secundair onderwijs in kaart gebracht: Een systematische literatuurstudie. $=$ Effectiveness of grade retention in primary and secondary education: A systematic review. Pedagogische Studiën, 90(5), 17-30. doi:10.1037/0033-. 295X.84.2.191

Grobler, A. A., Knight, M. R., Lens, W., \& Lacante, M. (2014). Motivational predictors of successful transition from grades 11 to 12 in South Africa. European Journal of Psychology of Education, 29(4), 693-709. doi:10.1007/s10212-014-0223-8

Haight, C., Kearney, C. A., Hendron, M., \& Schafer, R. (2011). Confirmatory analyses of the School Refusal Assessment Scale-Revised: Replication and extension to a truancy sample. Journal of Psychopathology and Behavioral Assessment, 33(2), 196-204. doi:10.1007/s10862-011-9218-9

Hakkarainen, A. M., Holopainen, L. K., \& Savolainen, H. K. (2015). A five-year follow-up on the role of educational support in preventing dropout from upper secondary education in Finland. Journal of Learning Disabilities, 48(4), 408-421. doi:10.1177/0022219413507603

Hammond, C., Linton, D., Smink, J., \& Drew, S. (2007). Dropout risk factors and exemplary programs: A technical report.

Hawkins, R. L., Jaccard, J., \& Needle, E. (2013). Nonacademic factors associated with dropping out of high school: Adolescent problem behaviors. Journal of the Society for Social Work and Research, 4(2), 58-75. doi:10.5243/jsswr.2013.5

Hodis, F. A., Meyer, L. H., McClure, J., Weir, K. F., \& Walkey, F. H. (2011). A longitudinal investigation of motivation and secondary school achievement using growth mixture modeling. Journal of Educational Psychology, 103(2), 312-323. doi:10.1037/a0022547

Inglés, C. J., Gonzálvez-Maciá, C., García-Fernández, J. M., Vicent, M., \& MartínezMonteagudo, M. C. (2015). Current status of research on school refusal. European Journal of Education and Psychology, 8(1), 37-52. doi:10.1016/j.ejeps.2015.10.005

Ingul, J. M., Klöckner, C. A., Silverman, W. K., \& Nordahl, H. M. (2012). Adolescent school absenteeism: Modelling social and individual risk factors. Child and Adolescent Mental Health, 17(2), 93-100. doi:10.1111/j.1475-3588.2011.00615.x

Ingul, J. M., \& Nordahl, H. M. (2013). Anxiety as a risk factor for school absenteeism: What differentiates anxious school attenders from non-attenders? Annals of General Psychiatry, 12.

Janosz, M. (2000). L'abandon scolaire chez les adolescents : perspective nord-américaine. Ville Ecole Intégration Enjeux, 122, 105-127. 
Janosz, M., Archambault, I., Morizot, J., \& Pagani, L. S. (2008). School engagement trajectories and their differential predictive relations to dropout. Journal of Social Issues, 64(1), 21-40. doi:10.1111/j.1540-4560.2008.00546.x

Janosz, M., \& Le Blanc, M. (1997). Les décrocheurs potentiels au secondaire : prévalence, facteurs de risque et dépistage. P.R.I.S.M.E. Psychiatrie, recherche et intervention en santé mentale de l'enfant, 7(2), 290-309.

Janosz, M., Le Blanc, M., Boulerice, B., \& Tremblay, R. E. (2000). Predicting different types of school dropouts : a typological approach with two longitudinal samples. Journal of Educational Psychology, 92(1), 171-190. doi:10.1037/0022-0663.92.1.171

Jimerson, S., Egeland, B., Sroufe, L. A., \& Carlson, B. (2000). A Prospective Longitudinal Study of High School Dropouts Examining Multiple Predictors Across Development. Journal of School Psychology, 38(6), 525-549. doi:10.1016/S0022-4405(00)00051-0

Jung, J., Krahé, B., \& Busching, R. (2017). Differential Risk Profiles for Reactive and Proactive Aggression. Social Psychology. doi:10.1037/0033-295X.108.1.273

Kearney, C. A. (2008). School absenteeism and school refusal behavior in youth: A contemporary review. Clinical Psychology Review, 28(3), 451-471. doi:10.1016/j.cpr.2007.07.012

Kearney, C. A., \& Graczyk, P. (2014). A response to intervention model to promote school attendance and decrease school absenteeism. Child \& Youth Care Forum, 43(1), 1-25. doi:10.1007/s10566-013-9222-1

Kim, K. H., \& Hull, M. F. (2012). Creative personality and anticreative environment for high school dropouts. Creativity Research Journal, 24(2-3), 169-176. doi:10.1080/10400419.2012.677318

Kindermann, T. A. (1993). Natural peer groups as contexts for individual development: The case of children's motivation in school. Developmental Psychology, 29(6), 970. doi: 10.1037/0012-1649.29.6.970

King, N. J., \& Bernstein, G. A. (2001). School refusal in children and adolescents: A review of the past 10 years. Journal of the American Academy of Child \& Adolescent Psychiatry, 40(2), 197-205. doi:10.1097/00004583-200102000-00014

Knollmann, M., Reissner, V., Kiessling, S., \& Hebebrand, J. (2013). Differentielle klassifikation schulvermeidenden verhaltens: Eine clusteranalytische untersuchung. = The differential classification of school avoidance: A cluster-analytic investigation. Zeitschrift für Kinder- und Jugendpsychiatrie und Psychotherapie, 41(5), 335-345. doi:10.1024/1422-4917//a000248

Kronick, R. F., \& Hargis, C. H. (1998). Dropouts: Who Drops Out and Why--And the Recommended Action. Second Edition.

Lamote, C., Speybroeck, S., Van Den Noortgate, W., \& Van Damme, J. (2013). Different pathways towards dropout: the role of engagement in early school leaving. Oxford Review of Education, 39(6), 739-760. doi:10.1080/03054985.2013.854202

Lamote, C., van Damme, J., van Den Noortgate, W., Speybroeck, S., Boonen, T., \& de Bilde, J. (2013). Dropout in secondary education: An application of a multilevel discrete-time hazard model accounting for school changes. Quality \& Quantity: International Journal of Methodology, 47(5), 2425-2446. doi:10.1007/s11135-012-9662-y

Lanson, A., \& Marcotte, D. (2012). Relations entre les symptômes dépressifs, la perception du soutien social et les distorsions cognitives des adolescents dans le contexte de la transition primaire-secondaire. Canadian Journal of Behavioural Science/Revue canadienne des sciences du comportement, 44(3), 231. 
Lee, J., Puig, A., \& Lee, S. M. (2012). The effect of the demand control and effort reward imbalance models on the academic burnout of Korean adolescents. Asia Pacific Journal of Education, 32(1), 113-123. doi:10.1080/02188791.2012.655373

Legleye, S., Obradovic, I., Janssen, E., Spilka, S., Le Nézet, O., \& Beck, F. (2010). Influence of cannabis use trajectories, grade repetition and family background on the schooldropout rate at the age of 17 years in France. European Journal of Public Health, 20(2), 157-163. doi:10.1093/eurpub/ckp148

Lehr, C. A., Hansen, A., Sinclair, M. F., \& Christenson, S. L. (2003). Moving Beyond Dropout Towards School Completion: An Integrative Review of Data-Based Interventions. School Psychology Review, 32(3), 342-364.

Lessard, A., Butler-Kisber, L., Fortin, L., \& Marcotte, D. (2014). Analyzing the discourse of dropouts and resilient students. The Journal of Educational Research, 107(2), 103-110. doi:10.1080/00220671.2012.753857

Lessard, A., Poirier, M., \& Fortin, L. (2010). Student-teacher relationship: A protective factor against school dropout? Procedia - Social and Behavioral Sciences, 2, 1636-1643. doi:10.1016/j.sbspro.2010.03.250

Liu, J., Zhao, S., Chen, X., Falk, E., \& Albarracín, D. (2017). The influence of peer behavior as a function of social and cultural closeness: A meta-analysis of normative influence on adolescent smoking initiation and continuation. Psychological bulletin, 143(10), 1082. doi:10.1037/bul0000113

Lovelace, M. D., Reschly, A. L., Appleton, J. J., \& Lutz, M. E. (2014). Concurrent and predictive validity of the student engagement instrument. Journal of Psychoeducational Assessment, 32(6), 509-520. doi:10.1177/0734282914527548

Lucio, R., Hunt, E., \& Bornovalova, M. (2012). Identifying the necessary and sufficient number of risk factors for predicting academic failure. Developmental Psychology, 48(2), 422-428. doi:10.1037/a0025939

Marcelli, D., \& Braconnier, A. (2008). Psychopathologie de l'adolescent (7 ed.). Paris: Masson.

Marcelli, D., \& Mezange, F. (2000). Les accidents à répétition chez l'adolescent. Traits anxieux, dépressifs et conduites de risque associés. Neuropsychiatrie de l'Enfance et de l'Adolescence, 48(3), 163-174.

Maric, M., Heyne, D. A., MacKinnon, D. P., van Widenfelt, B. M., \& Westenberg, P. M. (2013). Cognitive mediation of cognitive-behavioural therapy outcomes for anxietybased school refusal. Behavioural and Cognitive Psychotherapy, 41(5), 549-564. doi:10.1017/s1352465812000756

Markussen, E., Frøseth, M. W., \& Sandberg, N. (2011). Reaching for the unreachable: Identifying factors predicting early school leaving and non-completion in Norwegian upper secondary education. Scandinavian Journal of Educational Research, 55(3), 225253. doi:10.1080/00313831.2011.576876

Martin, A. J. (2012). Part II commentary: Motivation and engagement: Conceptual, operational, and empirical clarity. In S. L. Christenson, A. L. Reschly, \& C. Wylie (Eds.), Handbook of research on student engagement. (pp. 303-311). New York, NY, US: Springer Science + Business Media.

McLeod, J. D., Uemura, R., \& Rohrman, S. (2012). Adolescent mental health, behavior problems, and academic achievement. Journal of Health and Social Behavior, 53(4), 482-497. doi:10.1177/0022146512462888

Melkevik, O., Nilsen, W., Evensen, M., Reneflot, A., \& Mykletun, A. (2016). Internalizing Disorders as Risk Factors for Early School Leaving: A Systematic Review. Adolescent Research Review, 1-11. 
Meuret, D., \& Morlaix, S. (2006). L'influence de l'origine sociale sur les performances scolaires: par où passe-t-elle ? Revue française de sociologie, 47(1), 49-79. doi: $10.3917 /$ rfs.471.0049

Meylan, N., Doudin, P. A., Curchod-Ruedi, D., \& Stephan, P. (2015). Burnout scolaire et soutien social : l'importance du soutien des parents et des enseignants. Psychologie française, 60(1), 1-15. doi:10.1016/j.psfr.2014.01.003

Migali, G., \& Zucchelli, E. (2017). Personality traits, forgone health care and high school dropout: Evidence from US adolescents. Journal of Economic Psychology, 62, 98-119. doi:10.1016/j.joep.2017.06.007

Moulin, S., Doray, P., Prévost, J.-G., \& Delavictoire, Q. (2014). La propagation internationale d'une représentation. Le cas du décrochage scolaire. Histoire \& mesure, 29(1), 139-166.

OECD. (2016). Low-Performing Students: Why They Fall Behind and How To Help Them Succeed. Paris: OECD Publishing.

Orpinas, P., Horne, A. M., Song, X., Reeves, P. M., \& Hsieh, H. L. (2013). Dating trajectories from middle to high school: Association with academic performance and drug use. Journal of Research on Adolescence, 23(4), 772-784. doi:10.1111/jora.12029

Orpinas, P., \& Raczynski, K. (2016). School climate associated with school dropout among tenth graders. Pensamiento Psicológico, 14(1), 9-20. doi:10.11144/Javerianacali.PPSI14-1.scsd

Orpinas, P., Raczynski, K., Peters, J. W., Colman, L., \& Bandalos, D. (2015). Latent profile analysis of sixth graders based on teacher ratings: Association with school dropout. School Psychology Quarterly, 30(4), 577-592. doi:10.1037/spq0000107

Pagani, L. S., Brière, F. N., \& Janosz, M. (2017). Fluid reasoning skills at the high school transition predict subsequent dropout. Intelligence, 62, 48-53. doi:10.1016/j.intell.2017.02.006

Parker, P. D., \& Salmela-Aro, K. (2011). Developmental processes in school burnout: A comparison of major developmental models. Learning and Individual Differences, 21(2), 244-248. doi:10.1016/j.lindif.2011.01.005

Parlement Européen. (2009). Conclusions du Conseil de l'Union européenne du 12 mai 2009, Cadre stratégique pour la coopération européenne dans le domaine de l'éducation et de la formation, Éducation et formation 2020. Bruxelles.

Pharris-Ciurej, N., Hirschman, C., \& Willhoft, J. (2012). The 9th grade shock and the high school dropout crisis. Social Science Research, 41(3), 709-730. doi:10.1016/j.ssresearch.2011.11.014

Quiroga, C. V., Janosz, M., Bisset, S., \& Morin, A. J. S. (2013). Early adolescent depression symptoms and school dropout: Mediating processes involving self-reported academic competence and achievement. Journal of Educational Psychology, 105(2), 552-560. doi:10.1037/a0031524

Quiroga, C. V., Janosz, M., Lyons, J. S., \& Morin, A. J. S. (2012). Grade retention and seventh-grade depression symptoms in the course of school dropout among high-risk adolescents. Psychology, 3(9A), 749-755. doi:10.4236/psych.2012.329113

Ricard, N. C., \& Pelletier, L. G. (2016). Dropping out of high school: The role of parent and teacher self-determination support, reciprocal friendships and academic motivation. Contemporary Educational Psychology, 44-45, 32-40.

doi:10.1016/j.cedpsych.2015.12.003 
Richard, R., \& Marcotte, D. (2013). La relation temporelle entre l'anxiété et la dépression dans le contexte de la transition primaire-secondaire. Sante Mentale Au Quebec, 38(2), 257-275. doi:10.7202/1023999ar

Rumberger, R. W. (1987). High school dropouts: A review of issues and evidence. Review of Educational Research, 57(2), 101-121. doi:10.3102/00346543057002101

Rumberger, R. W. (1995). Dropping out of middle school: A multilevel analysis of students and schools. American educational Research journal, 32(3), 583-625.

Rumberger, R. W., \& Lim, S. A. (2008). Why students drop out of school: A review of 25 years of research. Retrieved from http://cdrp.ucsb.edu/dropouts/pubs_reports.htm

Rumberger, R. W., \& Palardy, G. J. (2004). Multilevel models for school effectiveness research. Handbook of quantitative methodology for the social sciences, 235-258.

Ryan, A. M. (2000). Peer groups as a context for the socialization of adolescents' motivation, engagement, and achievement in school. Educational Psychologist, 35(2), 101-111. doi:10.1207/S15326985EP3502_4

Ryan, A. M. (2001). The peer group as a context for the development of young adolescent motivation and achievement. Child Development, 72(4), 1135-1150.

Sibeoni, J., Moscoso, A., \& Revah-Levy, A. (2016). School refusal : Idiom of distress and/or Babel tower? European Psychiatry, 33, S444. doi:10.1016/j.eurpsy.2016.01.1285

Theunissen, M.-J., de Man, I., Verdonk, P., Bosma, H., \& Feron, F. (2015). Are Barbie and Ken too cool for school? A case-control study on the relation between gender and dropout. European Journal of Public Health, 25(1), 57-62. doi:10.1093/eurpub/cku097

Walburg, V. (2014). Burnout among high school students: A literature review. Children and youth services review, 42(Supplement C), 28-33. doi:10.1016/j.childyouth.2014.03.020

Wang, M., \& Fredricks, J. A. (2014). The reciprocal links between school engagement, youth problem behaviors, and school dropout during adolescence. Child Development, 85(2), 722-737. doi:10.1111/cdev.12138

Wang, M., \& Peck, S. C. (2013). Adolescent educational success and mental health vary across school engagement profiles. Developmental Psychology, 49(7), 1266-1276. doi: $10.1037 / \mathrm{a} 0030028$

Warren, J. R., \& Lee, J. C. (2003). The impact of adolescent employment on high school dropout: Differences by individual and labor-market characteristics. Social Science Research, 32(1), 98-128. doi:10.1016/S0049-089X(02)00021-2

Weitzman, M., Klerman, L. V., Lamb, G., Menary, J., \& Alpert, J. J. (1982). School absence: a problem for the pediatrician. Pediatrics, 69(6), 739-746. doi:10.1097/00004583198211000-00027

Weixler, F., \& Soudoplatoff, A.-S. (2015). Nouveau plan de lutte contre le décrochage scolaire. Les Cahiers Dynamiques(1), 16-25. doi:10.3917/1cd.063.0016

Wentzel, K. R. (1998). Social relationships and motivation in middle school: The role of parents, teachers, and peers. Journal of Educational Psychology, 90(2), 202. doi:10.1037/0022-0663.90.2.202

Wirthwein, L., Sparfeldt, J. R., Pinquart, M., Wegerer, J., \& Steinmayr, R. (2013). Achievement goals and academic achievement: A closer look at moderating factors. Educational Research Review, 10, 66-89. doi:10.1016/j.edurev.2013.07.001

Wood, L., Kiperman, S., Esch, R. C., Leroux, A. J., \& Truscott, S. D. (2017). Predicting dropout using student-and school-level factors: An ecological perspective. School Psychology Quarterly, 32(1), 35. doi:10.1037/spq0000152 


\section{Figure 1}

\section{Diagramme des flux.}

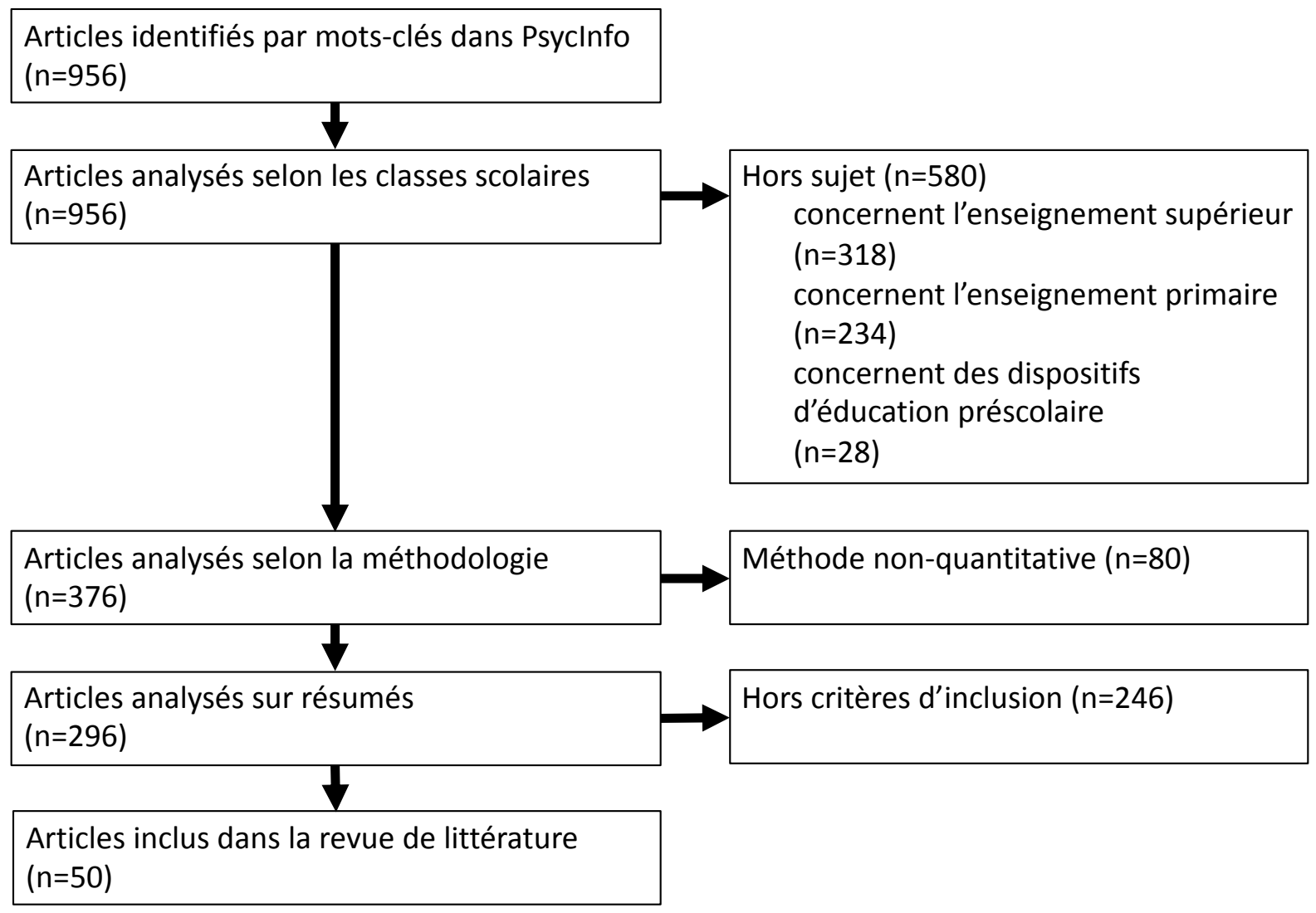




\section{Tableau 1}

Tableau des caractéristiques de la littérature 2010-2017.

\begin{tabular}{|c|c|c|c|c|c|}
\hline Articles & $\begin{array}{l}\text { Taille } \\
\text { échantillon } \\
\text { Approche }\end{array}$ & $\begin{array}{l}\text { Classes } \\
\text { d'âge } \\
\text { scolaires }\end{array}$ & $\begin{array}{l}\text { Définition des } \\
\text { décrocheurs }\end{array}$ & $\begin{array}{c}\text { Tests et } \\
\text { instruments de mesure }\end{array}$ & $\begin{array}{l}\text { Déterminants } \\
\text { les plus significatifs }\end{array}$ \\
\hline $\begin{array}{l}\text { Abar, } 2012 \\
\text { US }\end{array}$ & $\begin{array}{l}\mathrm{N}=14240 \\
\text { Longitudinale }\end{array}$ & 10th-12th & $\begin{array}{l}\text { Elèves non-inscrits en } \\
\text { dernière année (ne prend } \\
\text { pas en compte ceux ayant } \\
\text { obtenu le GED, redoublé, } \\
\text { décédé ou quitté le pays) }\end{array}$ & $\begin{array}{l}\text { Self-Determination Theory (SDT) ; Parent's } \\
\text { questionnaire; Administrator's } \\
\text { questionnaire; Student's questionnaire of } \\
\text { motivation items ; Math/Reading test }\end{array}$ & $\begin{array}{l}\text { Motivation externe ; Motivation } \\
\text { identifiée/introjectée; Motivation } \\
\text { identifiée/introjectée - externe }\end{array}$ \\
\hline $\begin{array}{l}\text { Bardou, } \\
2012 \\
\text { FR }\end{array}$ & $\mathrm{N}=450$ & $\begin{array}{l}6^{\mathrm{e}}-3^{\mathrm{e}} \\
(6 \text { th-9th) }\end{array}$ & $\begin{array}{l}\text { Elèves à risque (résultats } \\
\text { EMS et résultats scolaires) }\end{array}$ & $\begin{array}{l}\text { Echelle de Mobilisation Scolaire (EMS) ; } \\
\text { Echelle Toulousaine d'Estime de Soi ; } \\
\text { Moyenne générale annuelle }\end{array}$ & $\begin{array}{l}\text { Faible estime de soi socio-émotionnel } \\
\text { Faible estime de soi scolaire }\end{array}$ \\
\hline $\begin{array}{l}\text { Blaya, } 2011 \\
\text { FR, CA }\end{array}$ & $\mathrm{N}=963$ & $\begin{array}{l}4^{e}-3^{e} \\
(8 \text { th-9th) }\end{array}$ & $\begin{array}{l}\text { Elèves à risque (résultats } \\
\text { DEMS) }\end{array}$ & $\begin{array}{l}\text { Questionnaire d'évaluation du risque de } \\
\text { décrochage scolaire en milieu scolaire } \\
\text { (DEMS) ; Family Assessment Device ; } \\
\text { Classroom Environment Scale ; Child } \\
\text { Behavior Checklist ; } \\
\text { Behavior Assessment System for Children ; } \\
\text { Beck Depression Inventory }\end{array}$ & $\begin{array}{l}\text { Âge supérieur à la moyenne ; Attitudes } \\
\text { négatives envers l'école ; Problème } \\
\text { d'attention }\end{array}$ \\
\hline $\begin{array}{l}\text { Borgna, } \\
2016 \\
\text { IT }\end{array}$ & $\begin{array}{l}\mathrm{N}=6541 \\
\text { Rétrospective }\end{array}$ & $18-20$ ans & $\begin{array}{l}\text { Elèves ayant quitté l'école } \\
\text { sans diplôme }\end{array}$ & $\begin{array}{l}\text { Genre items ; Highest Parental Education } \\
\text { items ; Previous Achievement items }\end{array}$ & Genre masculin \\
\hline $\begin{array}{l}\text { Bowers, } \\
2012 \mathrm{a} \\
\text { US }\end{array}$ & $\begin{array}{l}\mathrm{N}=5400 \\
\text { Longitudinale }\end{array}$ & 9th-12h & $\begin{array}{l}\text { Elèves n'ayant pas obtenu } \\
\text { de diplôme à } 20 \text { ans } \\
\text { (comprend aussi ceux ayant } \\
\text { obtenu le GED) }\end{array}$ & $\begin{array}{l}\text { Student Background items ; Student } \\
\text { Behavior items ; School Context data }\end{array}$ & $\begin{array}{l}\text { Mauvaises performances scolaires; } \\
\text { Présence de gangs dans l'école ; } \\
\text { Croyances négatives sur la possibilité de } \\
\text { se faire des amis à l'école ; Projet de } \\
\text { cursus scolaire court }\end{array}$ \\
\hline
\end{tabular}




\begin{tabular}{|c|c|c|c|c|c|}
\hline $\begin{array}{l}\text { Bowers, } \\
2012 b \\
\text { US }\end{array}$ & $\begin{array}{l}\mathrm{N}=1470 \\
\text { Longitudinale }\end{array}$ & 9 th-12h & $\begin{array}{l}\text { Elèves n'ayant pas obtenu } \\
\text { de diplôme à } 20 \text { ans } \\
\text { (comprend aussi ceux ayant } \\
\text { obtenu le GED) }\end{array}$ & $\begin{array}{l}17 \text { independent variables ; cumulative GPA ; } \\
\text { Math/Reading test }\end{array}$ & $\begin{array}{l}\text { Attitudes négatives envers l'école } \\
\text { Difficultés scolaires régulières ; } \\
\text { Mauvaises performances scolaires }\end{array}$ \\
\hline $\begin{array}{l}\text { Breslau, } \\
2011 \\
\text { US }\end{array}$ & $\begin{array}{l}\mathrm{N}=29662 \\
\text { Rétrospective }\end{array}$ & $\begin{array}{l}18 \text { ans et } \\
\text { plus }\end{array}$ & $\begin{array}{l}\text { Elèves n'ayant pas obtenu } \\
\text { de diplôme du secondaire } \\
\text { et ayant passé moins de } 12 \\
\text { ans à l'école (ne prend pas } \\
\text { en compte ceux ayant } \\
\text { obtenu le GED) }\end{array}$ & DSM-IV & $\begin{array}{l}\text { Dépression/Dysthymie ; Episode } \\
\text { maniaque ; Trouble panique ; Phobie } \\
\text { social ; Phobie spécifique ; SSPT ; TAG ; } \\
\text { Trouble des conduites ; TDAH de type } \\
\text { inattentif ; TDAH de type hyperactif ; } \\
\text { TDAH de type combiné ; Consommation } \\
\text { de tabac ; Consommation d'alcool ; } \\
\text { Consommation d'une drogue illégale ; } \\
\text { Dépendance au tabac ; Dépendance à une } \\
\text { drogue illégale }\end{array}$ \\
\hline $\begin{array}{l}\text { Casillas, } \\
2012 \\
\text { US }\end{array}$ & $\mathrm{N}=3757$ & 7th-8th & $\begin{array}{l}\text { Elèves à risque (niveau } \\
\text { GPA) }\end{array}$ & EXPLORE scales ; ENGAGE scales & $\begin{array}{l}\text { Mauvaises performances scolaires } \\
\text { antérieures ; Mauvaises performances } \\
\text { scolaires ; Devoirs à la maison non } \\
\text { effectués ; Indiscipline scolaire ; Faible } \\
\text { comportement méthodique ; Genre } \\
\text { masculin }\end{array}$ \\
\hline $\begin{array}{l}\text { Cornell, } \\
2013 \\
\text { US }\end{array}$ & $\begin{array}{l}\mathrm{N}=2764 \\
\text { Longitudinale }\end{array}$ & 9th-12th & $\begin{array}{l}\text { Elèves ayant quitté le } \\
\text { cursus en cours, et qui ne } \\
\text { sont pas redoublants ou } \\
\text { réinscrits dans un autre } \\
\text { établissement }\end{array}$ & $\begin{array}{l}\text { Student questionnaire (Bullying items); } \\
\text { Teacher questionnaire (Bullying items); } \\
\text { Prevalence of Teasing and Bullying scale } \\
\text { Student/Teacher reported (PTB-S / PTB-T) ; } \\
\text { Math/English/Science/History test }\end{array}$ & $\begin{array}{l}\text { Expérience de bullying ; Établissement } \\
\text { scolaire de grande taille ; Mauvaises } \\
\text { performances scolaires ; Faible revenu } \\
\text { familial ; Appartenance à une minorité } \\
\text { ethnique }\end{array}$ \\
\hline $\begin{array}{l}\text { Delfabbro, } \\
2017 \\
\text { AU }\end{array}$ & $\begin{array}{l}\mathrm{N}=2552 \\
\text { Longitudinale }\end{array}$ & 10th-12th & $\begin{array}{l}\text { Elèves ayant quitté l'école } \\
\text { au moment d'une passation }\end{array}$ & $\begin{array}{l}\text { Negative Mood Scale ; Family Assessment } \\
\text { Device - General Functioning Scale; Life } \\
\text { Satisfaction Scale-Abbreviated Version ; } \\
\text { Rosenberg Self-Esteem Scale; General } \\
\text { Health Questionnaire-12; Demographic } \\
\text { items; Risky Health-Related Behaviors } \\
\text { items }\end{array}$ & $\begin{array}{l}\text { Age supérieur à la moyenne ; Mauvaise } \\
\text { santé physique }\end{array}$ \\
\hline
\end{tabular}




\begin{tabular}{|c|c|c|c|c|c|}
\hline $\begin{array}{l}\text { Eicher, } 2014 \\
\text { CH }\end{array}$ & $\begin{array}{l}\mathrm{N}=4312 \\
\text { Longitudinale }\end{array}$ & $\begin{array}{l}1^{\text {ère }}-4^{\mathrm{e}} \\
(10 \text { th- } \\
13 \text { th })\end{array}$ & $\begin{array}{l}\text { Elèves rapportant avoir } \\
\text { quitté une fois ou plus un } \\
\text { cursus avant son terme } \\
\text { Elèves à risque (intention } \\
\text { de décrocher) }\end{array}$ & $\begin{array}{l}\text { Education items ; Optimism items ; } \\
\text { Educational Stress scale ; Dropout Intentions } \\
\text { item }\end{array}$ & Intention de décrocher \\
\hline $\begin{array}{l}\text { Fall, } 2012 \\
\text { US }\end{array}$ & $\begin{array}{l}\mathrm{N}=14781 \\
\text { Longitudinale }\end{array}$ & 10th-12th & $\begin{array}{l}\text { Elèves non-inscrits en } \\
\text { dernière année et ayant } \\
\text { cumulés au moins } 4 \\
\text { semaines d'absences } \\
\text { injustifiées (ne prend pas } \\
\text { en compte ceux ayant } \\
\text { obtenu le GED) }\end{array}$ & $\begin{array}{l}\text { Self-System Model of Motivational } \\
\text { Development (SSMMD) ; Parent support } \\
\text { items ; Teacher support items ; Perceived } \\
\text { control items ; Perceived identification with } \\
\text { school items ; School engagement items ; } \\
\text { Math/Reading test }\end{array}$ & $\begin{array}{l}\text { Faible engagement comportemental } \\
\text { Faible engagement académique } \\
\text { Mauvaises performances scolaires }\end{array}$ \\
\hline $\begin{array}{l}\text { Fan, } 2012 \\
\text { US }\end{array}$ & $\begin{array}{l}\mathrm{N}=16194 \\
\text { Longitudinale }\end{array}$ & 10th-12th & $\begin{array}{l}\text { Elèves non-inscrits en } \\
\text { dernière année }\end{array}$ & $\begin{array}{l}\text { Expectancy-Values Model of Motivation } \\
\text { (EVMM) ; Scale of Intrinsic Versus } \\
\text { Extrinsic Orientation ; Motivated Strategies } \\
\text { for Learning Questionnaire ; Math/English } \\
\text { test }\end{array}$ & $\begin{array}{l}\text { Faibles attentes vis-à-vis des études ; } \\
\text { Faible revenu familial ; Appartenance à } \\
\text { une minorité ethnique }\end{array}$ \\
\hline $\begin{array}{l}\text { Fortin, } 2013 \\
\text { CA }\end{array}$ & $\begin{array}{l}\mathrm{N}=672 \\
\text { Longitudinale }\end{array}$ & $\begin{array}{l}6 e- \\
\text { DEC2 } \\
(6 \text { th-13h) }\end{array}$ & $\begin{array}{l}\text { Elèves ayant quitté l'école } \\
\text { et restant sans diplôme à } 19 \\
\text { ans }\end{array}$ & $\begin{array}{l}\text { Parental Participation in School Follow-Up } \\
\text { Measure ; Teacher's Attitudes Towards the } \\
\text { Student Scale ; Social Skills Rating System ; } \\
\text { Beck Depression Inventory ; Family } \\
\text { Environment Scale ; Classroom } \\
\text { Environment Scale }\end{array}$ & Mauvaises performances scolaires \\
\hline $\begin{array}{l}\text { Freeney, } \\
2012 \\
\text { IE }\end{array}$ & $\mathrm{N}=1311$ & $13-14$ ans & $\begin{array}{l}\text { Elèves à risque (intention } \\
\text { de décrocher) }\end{array}$ & $\begin{array}{l}\text { Theory of Planned Behavior (TPB); Student } \\
\text { Background items }\end{array}$ & Intention de décrocher \\
\hline $\begin{array}{l}\text { Frostad, } \\
2015 \\
\text { NO }\end{array}$ & $\mathrm{N}=2045$ & 16 ans & $\begin{array}{l}\text { Elèves à risque (intention } \\
\text { de décrocher) }\end{array}$ & $\begin{array}{l}\text { Teacher Support Scale ; Academic } \\
\text { Achievement Scale ; Parental Educational } \\
\text { Level Scale ; Perceived Peer Acceptance } \\
\text { Measure ; Social Participation Measure ; } \\
\text { Loneliness Scale ; Intention to Leave Scale }\end{array}$ & $\begin{array}{l}\text { Sentiment de solitude ; Faible soutien des } \\
\text { enseignants ; Mauvaises performances } \\
\text { scolaires ; Faible niveau de diplôme de la } \\
\text { mère }\end{array}$ \\
\hline
\end{tabular}




\begin{tabular}{|c|c|c|c|c|c|}
\hline $\begin{array}{l}\text { Gagné, } 2011 \\
\text { CA }\end{array}$ & $\begin{array}{l}\mathrm{N}=537 \\
\text { Longitudinale }\end{array}$ & $\begin{array}{l}1^{\text {ère }}-5^{\mathrm{e}} \\
(7 \text { th- } 11 \text { th })\end{array}$ & $\begin{array}{l}\text { Elèves non-inscrits en } \\
\text { dernière année et qui n'ont } \\
\text { pas obtenu de diplôme à } \\
\text { l'âge de } 20 \text { ans }\end{array}$ & $\begin{array}{l}\text { Beck Depression Inventory ; Classroom } \\
\text { Environment Scale ; Moyenne annuelle } \\
\text { Maths/Français }\end{array}$ & $\begin{array}{l}\text { Mauvaises performances scolaires des } \\
\text { élèves témoignant d'un état dépressif }\end{array}$ \\
\hline $\begin{array}{l}\text { Gagnon, } \\
2015 \\
\text { CA }\end{array}$ & $\mathrm{N}=1557$ & $14-18$ ans & $\begin{array}{l}\text { Elèves ayant quitté l'école } \\
\text { à la fin de l'année } \\
\text { d'enquête (y compris pour } \\
\text { une inscription en filière } \\
\text { professionnelle) }\end{array}$ & $\begin{array}{l}\text { Indice auto-rapporté de prédiction du } \\
\text { décrochage (IAR) ; Indice administratif (IA) } \\
\text { de prédiction du décrochage }\end{array}$ & $\begin{array}{l}\text { Attitudes négatives envers l'école ; Faible } \\
\text { portée du projet d'études }\end{array}$ \\
\hline $\begin{array}{l}\text { Grobler, } \\
2014 \\
\text { ZA }\end{array}$ & $\begin{array}{l}\mathrm{N}=1355 \\
\text { Longitudinale }\end{array}$ & 11th-12th & $\begin{array}{l}\text { Elèves échouant à valider } \\
\text { leur dernière année } \\
\text { d'études secondaires (12th) }\end{array}$ & $\begin{array}{l}\text { Self-Determination Theory (SDT); } \\
\text { Aspirations Index ; Learning and Study } \\
\text { Skills Inventory ; Academic self-regulation } \\
\text { Questionnaire ; Biographical Questionnaire } \\
\end{array}$ & Genre masculin \\
\hline $\begin{array}{l}\text { Haight, } 2011 \\
\text { US }\end{array}$ & $N=216$ & $11-17$ ans & $\begin{array}{l}\text { Elèves absentéistes placés } \\
\text { auprès de services sociaux }\end{array}$ & $\begin{array}{l}\text { School Refusal Assessment Scale-Child } \\
\text { (SRAS)/Parent-Revised }\end{array}$ & $\begin{array}{l}\text { Évitement de stimuli scolaires spécifiques } \\
\text {; Évitement de situations sociales } \\
\text { pénibles ; Anxiété de séparation ; } \\
\text { Renforcement d'occupations extra- } \\
\text { scolaires tangibles }\end{array}$ \\
\hline $\begin{array}{l}\text { Hakkarainen, } \\
2015 \\
\text { FI }\end{array}$ & $\begin{array}{l}\mathrm{N}=595 \\
\text { Longitudinale }\end{array}$ & 10th-12th & $\begin{array}{l}\text { Elèves n'ayant pas obtenu } \\
\text { de diplôme à } 21 \text { ans }\end{array}$ & $\begin{array}{l}\text { Error-Finding Test ; Word-Chain Test ; } \\
\text { Received Educational Support for Learning } \\
\text { Questionnaire ; Mathematical Skill Test }\end{array}$ & $\begin{array}{l}\text { Mauvaises performances en } \\
\text { mathématiques ; passage du } 9^{\text {th }} \text { Grade }\end{array}$ \\
\hline $\begin{array}{l}\text { Hawkins, } \\
2013 \\
\text { US }\end{array}$ & $\begin{array}{l}\mathrm{N}=3000 \\
\text { Longitudinale }\end{array}$ & 7th-12h & $\begin{array}{l}\text { Elèves rapportant ne pas } \\
\text { avoir obtenu de diplôme } \\
\text { (ne prend pas en compte } \\
\text { ceux ayant obtenu le GED) }\end{array}$ & $\begin{array}{l}\text { Empiric items of delinquency, marijuana } \\
\text { use, alcohol use, sexual activity, cigarette } \\
\text { smoking; GPA ; Academic aspirations items } \\
\text {; Perceived parental academic expectations } \\
\text { items ; Connectedness to school items; } \\
\text { Parent education items; Parental income et } \\
\text { welfare status items ; Demographic items }\end{array}$ & $\begin{array}{l}\text { Mauvaises performances scolaires ; Faible } \\
\text { niveau d'études de la mère ; } \\
\text { Consommation régulière de tabac ; } \\
\text { Activité sexuelle précoce ; Foyer familial } \\
\text { biparental ; Absence de projet d'études } \\
\text { supérieures }\end{array}$ \\
\hline $\begin{array}{l}\text { Hodis, } 2011 \\
\text { NZ }\end{array}$ & $\begin{array}{l}\mathrm{N}=1146 \\
\text { Longitudinale }\end{array}$ & \begin{tabular}{|l|}
11 th-13th \\
$(10$ th- \\
12 th $)$ \\
\end{tabular} & $\begin{array}{l}\text { Elèves à risque (nombre de } \\
\text { crédits obtenus chaque } \\
\text { année) }\end{array}$ & $\begin{array}{l}\text { Survey of National Certificate for } \\
\text { Educational Achievement (NCEA) Goals ; } \\
\text { Adaptive Living Scales, Doing My Best }\end{array}$ & $\begin{array}{l}\text { Genre masculin ; Comportement scolaire } \\
\text { basé sur le moindre effort }\end{array}$ \\
\hline
\end{tabular}




\begin{tabular}{|c|c|c|c|c|c|}
\hline & & & & $\begin{array}{l}\text { subscale ; Adaptive Living Scales, Doing } \\
\text { Just Enough subscale (DJE) }\end{array}$ & \\
\hline $\begin{array}{l}\text { Ingul, } 2012 \\
\text { NO }\end{array}$ & $\mathrm{N}=865$ & $\begin{array}{l}\text { VG1- } \\
\text { VG3 } \\
\text { (11th- } \\
13 \text { th) }\end{array}$ & $\begin{array}{l}\text { Elèves à risque } \\
\text { (absentéisme) }\end{array}$ & $\begin{array}{l}\text { Mood and Feeling Questionnaire ; IOWA } \\
\text { Personality Screen ; Strengths and } \\
\text { Difficulties Questionnaire ; Screen for Child } \\
\text { Anxiety Related Emotional Disorders }\end{array}$ & $\begin{array}{l}\text { Troubles externalisés ; Faible revenu } \\
\text { familial et mauvais accès aux soins }\end{array}$ \\
\hline $\begin{array}{l}\text { Ingul, } 2013 \\
\text { NO }\end{array}$ & $\mathrm{N}=865$ & $\begin{array}{l}\text { VG1- } \\
\text { VG3 } \\
\text { (11th- } \\
13 \text { th) }\end{array}$ & $\begin{array}{l}\text { Elèves à risque } \\
\text { (absentéisme) }\end{array}$ & $\begin{array}{l}\text { Iowa Personality Disorders Screen ; } \\
\text { Resilience Scale for Adolescents ; Life } \\
\text { Events Scale; Life Events Checklist ; Short } \\
\text { Mood and Feelings Questionnaire ; Kiddie } \\
\text { Schedule for Affective Disorders and } \\
\text { Schizophrenia ; Strengths and Difficulties } \\
\text { Questionnaire; Screen for Child Anxiety } \\
\text { Related Emotional Disorders }\end{array}$ & $\begin{array}{l}\text { Troubles psychiatriques / troubles de la } \\
\text { personnalité / problème relationnel ; } \\
\text { Consommation de drogues / problème } \\
\text { comportemental ; Résilience faible }\end{array}$ \\
\hline $\begin{array}{l}\text { Jung, } 2017 \\
\text { DE }\end{array}$ & $\begin{array}{l}\mathrm{N}=1479 \\
\text { Longitudinale }\end{array}$ & 9-20 ans & $\begin{array}{l}\text { Elèves ayant quitté l'école } \\
\text { au moment de la seconde } \\
\text { passation } \\
\text { Elèves à risque (niveau } \\
\text { d'évaluation scolaire) }\end{array}$ & $\begin{array}{l}\text { Instrument of Reactive and Proactive } \\
\text { Aggression; Strength and Difficulties } \\
\text { Questionnaire; Affiliation With Aggressive } \\
\text { Peers items (self-reported and teachers } \\
\text { ratings); Academic Failure (Grades in Math, } \\
\text { German, and English) }\end{array}$ & Genre masculin ; Agressivité proactive \\
\hline $\begin{array}{l}\text { Kim, } 2012 \\
\text { US }\end{array}$ & $\begin{array}{l}\mathrm{N}=42190 \\
\text { Longitudinale }\end{array}$ & 10th-12h & $\begin{array}{l}\text { Elèves ayant quitté le } \\
\text { cursus avant son terme et } \\
\text { non réinscrits l'année } \\
\text { suivante }\end{array}$ & $\begin{array}{l}\text { Scales for Rating the Behavioral } \\
\text { Characteristics of Superior Students - } \\
\text { Revised ; Torrance Tests of Creative } \\
\text { Thinking; Runco Ideational Behavior Scale } \\
\text {; Student questionnaire (Creativity items) }\end{array}$ & $\begin{array}{l}\text { Importante créativité ; Accord pour la } \\
\text { désobéissance aux règles scolaires ; } \\
\text { Sentiment d'être rabaissé par les } \\
\text { enseignants ; Investissement dans un } \\
\text { projet professionnel ; Pratique du } \\
\text { cheerleading; Importance du temps passé } \\
\text { entre domicile et école (oisiveté ou } \\
\text { activité de loisirs) }\end{array}$ \\
\hline $\begin{array}{l}\text { Knollmann, } \\
2013 \\
\text { GE } \\
\end{array}$ & $\mathrm{N}=169$ & $8-16$ ans & $\begin{array}{l}\text { Élèves refusant d'aller à } \\
\text { l'école }\end{array}$ & $\begin{array}{l}\text { Child Behavior Checklist ; Youth Self- } \\
\text { Report }\end{array}$ & $\begin{array}{l}\text { Combinaison de troubles externalisés et } \\
\text { internalisés ; hyper-anxiété }\end{array}$ \\
\hline
\end{tabular}




\begin{tabular}{|c|c|c|c|c|c|}
\hline $\begin{array}{l}\text { Lamote, } \\
\text { Van Damme } \\
2013 \\
\text { BE }\end{array}$ & $\begin{array}{l}\mathrm{N}=4735 \\
\text { Longitudinale }\end{array}$ & $\begin{array}{l}1^{\text {ère }}-6^{\mathrm{e}} \\
(7 \text { th-12th })\end{array}$ & $\begin{array}{l}\text { Elèves ayant quitté le } \\
\text { cursus avant son terme sans } \\
\text { diplôme }\end{array}$ & $\begin{array}{l}\text { Language Achievement Test; Getlov } \\
\text { Intelligence Test ; Math Achievement Test ; } \\
\text { Sociodemographic and academic items }\end{array}$ & $\begin{array}{l}\text { Passage du } 11^{\text {th }} \text { Grade } ; \text { Redoublement } \\
\text { Établissement situé en zone économique } \\
\text { et sociale défavorisée / mauvaises } \\
\text { relations entre élèves et enseignants }\end{array}$ \\
\hline $\begin{array}{l}\text { Lamote, } \\
\text { Speybroeck } \\
2013 \\
\text { BE }\end{array}$ & $\begin{array}{l}\mathrm{N}=4604 \\
\text { Longitudinale }\end{array}$ & $\begin{array}{l}1^{\text {ère }}-6^{\mathrm{e}} \\
(7 \text { th-12th })\end{array}$ & $\begin{array}{l}\text { Élèves ayant quitté le } \\
\text { cursus avant son terme sans } \\
\text { diplôme }\end{array}$ & $\begin{array}{l}\text { Relationship with teachers' scale; Attitude } \\
\text { toward homework' scale; Sociodemographic } \\
\text { and academic items }\end{array}$ & $\begin{array}{l}\text { Faible engagement émotionnel au début } \\
\text { du cursus secondaire }\end{array}$ \\
\hline $\begin{array}{l}\text { Lee, } 2012 \\
\text { KR }\end{array}$ & $\mathrm{N}=1530$ & $8-18$ ans & $\begin{array}{l}\text { Elèves à risque (résultats } \\
\text { des échelles de burnout) }\end{array}$ & $\begin{array}{l}\text { Korean Academic Burnout Inventory; } \\
\text { Academic Demand-Control Inventory; } \\
\text { Effort-Reward Inventory ; Maslach Burnout } \\
\text { Inventory-Student Survey ; School Burnout } \\
\text { Inventory }\end{array}$ & $\begin{array}{l}\text { Croyance dans un faible rapport entre } \\
\text { efforts et récompenses scolaires }\end{array}$ \\
\hline $\begin{array}{l}\text { Legleye, } \\
2010 \\
\text { FR }\end{array}$ & $\begin{array}{l}\mathrm{N}=29393 \\
\text { Rétrospective }\end{array}$ & 17 ans & $\begin{array}{l}\text { Elèves déclarant n'être plus } \\
\text { scolarisées à } 17 \text { ans }\end{array}$ & Drugs use items ; Family items & $\begin{array}{l}\text { Parents séparés ; Parents inemployés ; } \\
\text { Parents exerçant un métier peu qualifié ; } \\
\text { Redoublement ; Consommation de } \\
\text { cannabis ; Au moins une expérience } \\
\text { d'intoxication à l'alcool ; Consommation } \\
\text { quotidienne de tabac ; Consommation } \\
\text { d'une ou deux drogues rares }\end{array}$ \\
\hline $\begin{array}{l}\text { Lessard, } \\
2010 \\
\text { CA }\end{array}$ & $\mathrm{N}=4312$ & $\begin{array}{l}1^{\text {ère }}-5^{\mathrm{e}} \\
(7 \text { th- } 11 \text { th })\end{array}$ & $\begin{array}{l}\text { Elèves à risque (résultats } \\
\text { DAT) }\end{array}$ & $\begin{array}{l}\text { Dropout Assessment Tool (DAT); } \\
\text { Classroom Environment Scale ; Student Life } \\
\text { Questionnaire }\end{array}$ & $\begin{array}{l}\text { Faible satisfaction scolaire (garçons); } \\
\text { Faible sentiment de réussite (garçons); } \\
\text { Faible satisfaction scolaire (filles) ; Faible } \\
\text { sentiment de réussite (filles) ; Faible } \\
\text { engagement scolaire (filles) }\end{array}$ \\
\hline $\begin{array}{l}\text { Lovelace, } \\
2014 \\
\text { US }\end{array}$ & $\mathrm{N}=11588$ & 9th & $\begin{array}{l}\text { Elèves ayant quitté le } \\
\text { cursus avant son terme sans } \\
\text { diplôme }\end{array}$ & Student Engagement Instrument (SEI) & $\begin{array}{l}\text { Faible soutien familial aux études ; Faible } \\
\text { contrôle sur le travail scolaire ; Faible } \\
\text { relations élèves-enseignants ; Faible } \\
\text { soutien des pairs aux études ; Absence de } \\
\text { projets et d'objectifs postérieurs }\end{array}$ \\
\hline $\begin{array}{l}\text { Lucio, } 2012 \\
\text { US }\end{array}$ & \begin{tabular}{l|}
$\mathrm{N}=14796$ \\
Longitudinale
\end{tabular} & 9th-12th & $\begin{array}{l}\text { Elèves à risque (niveau } \\
\text { GPA) }\end{array}$ & $\begin{array}{l}\text { Sociodemographic items ; } 12 \text { independent } \\
\text { variables ; cumulative GPA) }\end{array}$ & $\begin{array}{l}\text { Genre masculin ; Faible revenu familial ; } \\
\text { Appartenance à une minorité ethnique ; }\end{array}$ \\
\hline
\end{tabular}




\begin{tabular}{|c|c|c|c|c|c|}
\hline & & & & & $\begin{array}{l}\text { Devoirs à la maison mal effectués ; } \\
\text { Faibles attentes vis-à-vis de l'école ; } \\
\text { Comportement scolaire inadapté ; faible } \\
\text { confiance dans ses habilités scolaires ; } \\
\text { Faible engagement académique ; } \\
\text { Sentiment d'insécurité à l'école ; } \\
\text { Redoublement ; Absentéisme ; Mobilité } \\
\text { scolaire }\end{array}$ \\
\hline $\begin{array}{l}\text { Maric, } 2012 \\
\text { US }\end{array}$ & $\begin{array}{l}\mathrm{N}=231 \\
\mathrm{n}=50 \\
\text { (School } \\
\text { Refusers) } \\
\text { Transversale }\end{array}$ & $11-17$ ans & $\begin{array}{l}\text { Elèves en refus scolaire } \\
\text { anxieux }\end{array}$ & $\begin{array}{l}\text { Children's Negative Cognitive Error } \\
\text { Questionnaire-Revised ; Anxiety Disorders } \\
\text { Interview Schedule-Child/Parent ; } \\
\text { Children's Automatic Thoughts Scale- } \\
\text { Negative/Positive ; Multidimensional } \\
\text { Anxiety Scale for Children }\end{array}$ & $\begin{array}{l}\text { Pensées automatiques négatives sur les } \\
\text { difficultés scolaires personnelles }\end{array}$ \\
\hline $\begin{array}{l}\text { Markussen, } \\
2011 \\
\text { NO }\end{array}$ & $\begin{array}{l}\mathrm{N}=9749 \\
\text { Longitudinale }\end{array}$ & 10th-12th & $\begin{array}{l}\text { Elèves ayant quitté le } \\
\text { cursus avant son terme sans } \\
\text { diplôme } \\
\text { Elèves ayant atteint la } \\
\text { dernière année d'étude } \\
\text { mais n'ayant pas eu de } \\
\text { diplôme }\end{array}$ & $\begin{array}{l}16 \text { variables indépendantes } \\
\text { sociodémographiques, académiques et } \\
\text { psychologiques }\end{array}$ & $\begin{array}{l}\text { Faible engagement scolaire /mauvaises } \\
\text { performances scolaires précoces / milieu } \\
\text { familial défavorisé / genre masculin }\end{array}$ \\
\hline $\begin{array}{l}\text { Meylan, } \\
2015 \\
\text { CH }\end{array}$ & $\mathrm{N}=387$ & $13-17$ ans & $\begin{array}{l}\text { Elèves à risque (valeur } \\
\text { absentéisme et moyenne } \\
\text { notes) }\end{array}$ & $\begin{array}{l}\text { School Burnout Inventory ; Child and } \\
\text { Adolescent Social Support Scale (CASSS- } \\
\text { f) ; Variables académiques }\end{array}$ & Faible soutien perçu des parents \\
\hline $\begin{array}{l}\text { Migali, } 2017 \\
\text { US }\end{array}$ & $\begin{array}{l}\mathrm{N}=20745 \\
\text { Longitudinale }\end{array}$ & 7 th-12th & $\begin{array}{l}\text { Elèves déclarant avoir } \\
\text { décroché ou quitté l'école } \\
\text { sans diplôme (comprend } \\
\text { aussi ceux ayant obtenu le } \\
\text { GED) }\end{array}$ & $\begin{array}{l}\text { International Personality Item Pool ; Add } \\
\text { Health Picture Vocabulary Test; NEO } \\
\text { Personality Inventory ; Parent Questionnaire }\end{array}$ & Introversion ; Mauvaise hygiène de vie \\
\hline $\begin{array}{l}\text { Orpinas, } \\
2013 \\
\text { US }\end{array}$ & $\begin{array}{l}\mathrm{N}=620 \\
\text { Longitudinale }\end{array}$ & 6th-12th & $\begin{array}{l}\text { Elèves sans diplôme ayant } \\
\text { quitté le cursus en cours ou } \\
\text { ayant été expulsés, et non }\end{array}$ & $\begin{array}{l}\text { Assessment System for Children-Teacher } \\
\text { Rating Scales-Adolescent Version ; Problem }\end{array}$ & $\begin{array}{l}\text { Rendez-vous amoureux fréquents et } \\
\text { précoces }\end{array}$ \\
\hline
\end{tabular}




\begin{tabular}{|c|c|c|c|c|c|}
\hline & & & $\begin{array}{l}\text { réinscrits dans une autre } \\
\text { école }\end{array}$ & $\begin{array}{l}\text { Behavior Frequency Scales ; Computer- } \\
\text { Assisted Survey Interview }\end{array}$ & \\
\hline $\begin{array}{l}\text { Orpinas, } \\
2015 \\
\text { US }\end{array}$ & $\begin{array}{l}\mathrm{N}=675 \\
\text { Longitudinale }\end{array}$ & 6th-12th & $\begin{array}{l}\text { Elèves ne fréquentant plus } \\
\text { l'école à la fin de la } \\
\text { dernière année du } \\
\text { secondaire et n'ayant pas } \\
\text { obtenu de diplôme }\end{array}$ & $\begin{array}{l}\text { Behavior Assessment System for Children - } \\
\text { Teacher Rating Scales for adolescents ; } \\
\text { Problem Behavior Frequency Scales ; Life } \\
\text { Satisfaction Scale }\end{array}$ & $\begin{array}{l}\text { Troubles externalisés ; Troubles } \\
\text { internalisés ; faibles compétences } \\
\text { sociales ; Indiscipline scolaire récurrente }\end{array}$ \\
\hline $\begin{array}{l}\text { Orpinas, } \\
2016 \\
\text { US }\end{array}$ & $\begin{array}{l}\mathrm{N}=638 \\
\text { Longitudinale }\end{array}$ & 6th-12th & $\begin{array}{l}\text { Elèves sans diplôme ayant } \\
\text { quitté le cursus en cours ou } \\
\text { ayant été expulsés, et non } \\
\text { réinscrits dans une autre } \\
\text { école }\end{array}$ & $\begin{array}{l}\text { Empiric scales of Victimization and } \\
\text { Relationships in school }\end{array}$ & $\begin{array}{l}\text { Victimisation vis-à-vis des autres ; Faible } \\
\text { réseau relationnel à l'école ; Faible } \\
\text { recherche d'attention auprès des adultes } \\
\text { de l'école; Genre masculin }\end{array}$ \\
\hline $\begin{array}{l}\text { Pagani, } 2017 \\
\text { CA }\end{array}$ & $\begin{array}{l}\mathrm{N}=4962 \\
\text { Longitudinale }\end{array}$ & $\begin{array}{l}1^{\text {ère }}-5^{\mathrm{e}} \\
(7 \mathrm{th}-11 \mathrm{th})\end{array}$ & $\begin{array}{l}\text { Elèves ayant quitté le } \\
\text { cursus avant son terme sans } \\
\text { diplôme, et non réinscrits } \\
\text { dans les deux années } \\
\text { suivantes }\end{array}$ & $\begin{array}{l}\text { Raven Standard Progressive Matrices } \\
\text { (SPM) ; Socio-demographic and Academic } \\
\text { indicators ; Family Risk Factors ; Academic } \\
\text { Performance (self-reported Grades in Math } \\
\text { and French); School Engagement Scale; } \\
\text { Delinquency Scale }\end{array}$ & $\begin{array}{l}\text { Âge supérieur à la moyenne ; Genre } \\
\text { masculin ; Milieu familial à risque ; } \\
\text { Mesure d'intelligence plus faible que la } \\
\text { moyenne ; Appartenance à une minorité } \\
\text { ethnique; Délinquance ; Mauvaises } \\
\text { performances scolaires }\end{array}$ \\
\hline $\begin{array}{l}\text { Pharris, } \\
2012 \\
\text { US }\end{array}$ & $\begin{array}{l}\mathrm{N}=7441 \\
\text { Longitudinale }\end{array}$ & 8th-12th & $\begin{array}{l}\text { Elèves n'ayant pas obtenu } \\
\text { de diplôme au terme du } \\
\text { cursus normal (comprend } \\
\text { décrocheurs et redoublants) }\end{array}$ & $\begin{array}{l}\text { Genre; Ethnicity; Family income; } \\
\text { Neighborhood; Retention; GPA 8th/9th. }\end{array}$ & Passage du 9th grade \\
\hline $\begin{array}{l}\text { Quiroga, } \\
2012 \\
\text { CA }\end{array}$ & $\begin{array}{l}\mathrm{N}=453 \\
\text { Longitudinale }\end{array}$ & $\begin{array}{l}1^{\text {ère }}- \\
\text { DEC1 } \\
(7 \text { th-12th) }\end{array}$ & $\begin{array}{l}\text { Elèves ayant quitté le } \\
\text { cursus avant son terme sans } \\
\text { diplôme }\end{array}$ & $\begin{array}{l}\text { Inventory to Diagnose Depression ; Beck } \\
\text { Anxiety Inventory ; Student-Teacher } \\
\text { Relationship Scale; Diagnostic Interview } \\
\text { Schedule }\end{array}$ & $\begin{array}{l}\text { Redoublement ; Enseignement adapté ; } \\
\text { Fréquentation d'élèves en train de } \\
\text { décrocher Comportement rebelle ; } \\
\text { Symptômes de dépression au 7th grade ; } \\
\text { Mauvaise réussite scolaire ; Faible } \\
\text { compétence académique ; Faible } \\
\text { éducation parentale }\end{array}$ \\
\hline $\begin{array}{l}\text { Quiroga, } \\
2013 \\
\text { CA }\end{array}$ & $\begin{array}{l}\mathrm{N}=493 \\
\text { Longitudinale }\end{array}$ & $\begin{array}{l}1^{\text {ère }}- \\
\text { DEC1 } \\
(7 \text { th-12th) }\end{array}$ & $\begin{array}{l}\text { Elèves n'ayant pas obtenu } \\
\text { de diplôme (avant, au }\end{array}$ & $\begin{array}{l}\text { Inventory of Diagnose Depression ; Student } \\
\text { questionnaire ; Self-reported performance } \\
\text { Math/French }\end{array}$ & $\begin{array}{l}\text { Redoublement ; Faible éducation } \\
\text { parentale ; Faible compétence académique } \\
\text {; Mauvaises performances scolaires }\end{array}$ \\
\hline
\end{tabular}




\begin{tabular}{|c|c|c|c|c|c|}
\hline & & & $\begin{array}{l}\text { terme ou un an après la } \\
\text { dernière année du cursus) }\end{array}$ & & \\
\hline $\begin{array}{l}\text { Ricard, } 2016 \\
\text { CA }\end{array}$ & $\begin{array}{l}\mathrm{N}=624 \\
\text { Longitudinale }\end{array}$ & $\begin{array}{l}4^{\mathrm{e}}-\mathrm{DEC} 1 \\
(10 \text { th- } \\
12 \text { th })\end{array}$ & $\begin{array}{l}\text { Elèves sans diplôme ayant } \\
\text { rapporté avoir } \\
\text { définitivement quitté } \\
\text { l'école }\end{array}$ & $\begin{array}{l}\text { Self-Determination Theory (SDT) ; } \\
\text { Interpersonal Behavior Questionnaire-Short } \\
\text { Version ; Self-Determination Index ; } \\
\text { Academic Motivation Scale }\end{array}$ & $\begin{array}{l}\text { Faibles liens d'amitié réciproque ; Faible } \\
\text { support des parents pour les besoins } \\
\text { psychologiques de base }\end{array}$ \\
\hline $\begin{array}{l}\text { Theunissen, } \\
2015 \\
\text { NL }\end{array}$ & $\begin{array}{l}\mathrm{N}=660 \\
\mathrm{n}=330 \\
\text { (Dropouts) } \\
\text { Transversale } \\
\text { Retrospective }\end{array}$ & $18-23$ ans & $\begin{array}{l}\text { Elèves âgés de plus de } 18 \\
\text { ans et sans diplôme } \\
\text { déclarant avoir quitté } \\
\text { l'école } \\
\text { Elèves à risque (âgés de } \\
\text { plus de } 18 \text { ans et sans } \\
\text { diplôme déclarant être } \\
\text { encore à l'école) }\end{array}$ & $\begin{array}{l}\text { Adolescent Masculinity Ideology in } \\
\text { Relationships Scale (AMIRS); Adolescent } \\
\text { Femininity Ideology Scale (AFIS); Genre } \\
\text { identity items; Familial items ; Education } \\
\text { items }\end{array}$ & $\begin{array}{l}\text { Croyance modérée et élevée dans les } \\
\text { stéréotypes féminins ; Croyance élevée } \\
\text { dans les stéréotypes masculins }\end{array}$ \\
\hline $\begin{array}{l}\text { Wang, } 2014 \\
\text { US }\end{array}$ & $\begin{array}{l}\mathrm{N}=997 \\
\text { Longitudinale }\end{array}$ & 7th-12th & $\begin{array}{l}\text { Elèves non-inscrits en } \\
\text { dernière année et n'ayant } \\
\text { pas obtenu de diplôme } \\
\text { (comprend aussi ceux ayant } \\
\text { obtenu le GED) }\end{array}$ & $\begin{array}{l}\text { Monitoring the Future Questionnaire ; } \\
\text { Substance Use Frequency Scale ; School } \\
\text { Participation Scale ; School Identification } \\
\text { Scale ; Self-Regulated Learning Scale ; } \\
\text { Youth-Report Survey }\end{array}$ & $\begin{array}{l}\text { Mauvaises performances scolaires ; Faible } \\
\text { engagement comportemental ; Faible } \\
\text { engagement émotionnel ; Délinquance ; } \\
\text { Consommation d'alcool, tabac et cannabis }\end{array}$ \\
\hline $\begin{array}{l}\text { Wood, } 2017 \\
\text { US }\end{array}$ & $\mathrm{N}=14106$ & 11th & $\begin{array}{l}\text { Elèves déclarant avoir } \\
\text { quitté l'école sans diplôme } \\
\text { depuis } 2 \text { ans ou élèves sans } \\
\text { diplôme non-réinscrits } \\
\text { pendant } 3 \text { ans }\end{array}$ & $\begin{array}{l}\text { Socio-demographic, Academic, Parent and } \\
\text { School indicators; } \\
\text { Math test }\end{array}$ & $\begin{array}{l}\text { Redoublement ; Mauvaises performances } \\
\text { scolaires ; Genre masculin ; Établissement } \\
\text { scolaire de grande taille }\end{array}$ \\
\hline
\end{tabular}

La classe d'âge est indiquée selon les niveaux scolaires spécifiques au système scolaire concerné dans les études. Pour rendre la comparaison des données plus lisible, des niveaux scolaires standards sont indiqués entre parenthèse allant de 6th à 13th depuis la classe d'âge des $11 / 12$ ans (équivalente des $6^{\mathrm{e}}$ française et 6th américaine) à celle des 18/19 ans (soit une classe d'âge supplémentaire par rapport aux systèmes français et américain). L'amplitude d'âge est indiquée directement pour les études concernant des échantillons considérés hors niveau scolaire. 